\title{
STOCHASTIC FUNCTIONAL PARTIAL DIFFERENTIAL EQUATIONS: EXISTENCE, UNIQUENESS AND ASYMPTOTIC STABILITY
}

\author{
Tomás Caraballo \\ Dpto. Ecuaciones Diferenciales y Análisis Numérico. \\ Universidad de Sevilla. Apartado de Correos 1160. \\ 41080-SEVILLA, SPAIN \\ Email: caraball@numer.us.es \\ Kai LiU* Aubrey Truman \\ Department of Mathematics \\ University of Wales Swansea \\ Singleton Park, Swansea \\ SA2 8PP, UK \\ Email: k.liu@swansea.ac.uk
}

\begin{abstract}
Existence and uniqueness of strong solutions for a class of stochastic functional differential equations in Hilbert spaces are established. Sufficient conditions which guarantee the transference of mean square and pathwise exponential stability from stochastic partial differential equations to stochastic functional partial differential equations are studied. The stability results derived are also applied to stochastic ordinary differential equations with hereditary characteristics. In particular, as a direct consequence our main results improve some of those from Mao and Shah [13] in which it is proved that under certain conditions pathwise exponential stability is transferred from nondelay equations to delay ones if the constant time lag appearing in the problem is sufficiently small, while in our treatment the transference actually holds for arbitrary bounded delay variables not only in finite but in infinite dimensions.
\end{abstract}

Keywords: Stochastic partial differential equation; Stochastic functional partial differential equation; Mean square and pathwise exponential stability.

AMS 1991 Classifications: 60H15, 34K40.

* Author of Correspondence. 


\section{INTRODUCTION}

The study of stochastic functional differential equations is motivated by the fact that when one wants to model some evolution phenomena arising in Physics, Biology and Engineering etc., some hereditary characteristics such as aftereffect, time lag and time delay can appear in the variables (see, for example, Kolmanovskii and Myshkis [8], Mohammed [15]). On the other hand, one of the most important and interesting problems in the analysis of stochastic functional differential equations is their stability, the theory of which (mainly for finite dimensional systems) has been greatly developed over the last several years.

As is well known, in the case without any hereditary features, Lyapunov's technique is available to obtain sufficient conditions for the stability of solutions of stochastic (partial) differential equations. However, in the case of stochastic differential equations with hereditary properties, for instance, even with constant time delays, Lyapunov's method becomes difficult to apply effectively as Krasovskii [10] pointed out for the study of stability of ordinary differential equations, and as Kushner [11] and El'sgol'ts and Norkin [5] (among others) did for stochastic differential equations. The main reason is that it is much more difficult (or even impossible in some cases) to construct proper Lyapunov functions (or functionals) for stochastic functional differential equations than for those without any hereditary characteristics. As a consequence, a comparison technique has been developed by various authors such as Krasovskii [10] and Mao and Shah [13] (among others). Let us illustrate this point of our motivation in more detail.

Consider the following stochastic functional differential equation

$$
x(t)=\int_{0}^{t} f\left(s, x(s), x\left(s-h_{1}\right)\right) d s+\int_{0}^{t} \int_{-h_{2}}^{0} g(s, x(s+r)) h(r) d r d w(s), \quad t>0,
$$

where $h_{1}>0, h_{2}>0$, or equivalently,

$$
\begin{aligned}
x(t)= & \int_{0}^{t} f(s, x(s), x(s)) d s+\int_{0}^{t} g(s, x(s)) d w(s) \\
& +\int_{0}^{t}\left[f\left(s, x(s), x\left(s-h_{1}\right)\right)-f(s, x(s), x(s))\right] d s \\
& +\int_{0}^{t}\left[\int_{-h_{2}}^{0} g(s, x(s+r)) h(r) d r-g(s, x(s))\right] d w(s) .
\end{aligned}
$$

We can regard (1) as the perturbed system of the corresponding stochastic differential equation without hereditary characteristics

$$
x(t)=\int_{0}^{t} f(s, x(s), x(s)) d s+\int_{0}^{t} g(s, x(s)) d w(s), \quad t>0 .
$$

Clearly, if the time lag scales $h_{1}>0, h_{2}>0$ are sufficiently small, the perturbation term

$$
\int_{0}^{t}\left[f\left(s, x(s), x\left(s-h_{1}\right)\right)-f(s, x(s), x(s))\right] d s
$$




$$
+\int_{0}^{t}\left[\int_{-h_{2}}^{0} g(s, x(s+r)) h(r) d r-g(s, x(s))\right] d w(s),
$$

could be expected to be so small that the perturbed equation (1) would behave asymptotically as Equation (3) does. For instance, we could expect that if Equation (3) is exponentially stable and the time lags $h_{1}>0, h_{2}>0$ are small enough, then Equation (1) will remain exponentially stable. So, in order to find out whether the functional equation (1) is exponentially stable, one can check the exponential stability of the equation (3) and then compute whether the time lags $h_{1}>0, h_{2}>0$ are sufficiently small. In other words, the difficult problem of stability for functional equations would have been transferred to an easier one (the stability of equations without hereditary characteristics).

Motivated by the intuitive ideas described above, Mao and Shah [13] obtained some sufficient conditions for the $p$-th moment exponential stability (and also pathwise stability) of stochastic ordinary differential delay equations. For example, consider the following onedimensional stochastic delay differential equation

$$
d x(t)=f(t, x(t), x(t-h)) d t+g(t, x(t)) d w(t), \quad t>0,
$$

where $h>0$, or equivalently,

$$
\begin{aligned}
d x(t)= & f(t, x(t), x(t)) d t+g(t, x(t)) d w(t) \\
& +[f(t, x(t), x(t-h))-f(t, x(t), x(t))] d t .
\end{aligned}
$$

It was proved in [13] that under some circumstances pathwise exponential stability is transferred from the nondelay equation (i.e., $h=0$ in (5)) to the delay one (4) if the constant time lag $h>0$ appearing in the problem is sufficiently small.

Nevertheless, it is worth pointing out that the results derived in [13] are somewhat restrictive for many practical applications. In fact, the situation turns out to be rather complicated when one considers the general functional differential equations, even the usual stochastic delay differential systems. For instance, there exist a wide variety of interesting problems in which it is possible to ensure that if nondelay equations are exponentially stable, then delay ones remain exponentially stable whatever the delay interval could be, what is more even if, the delays are not constants. In this work, by a completely different approach from that in [13] we shall carry out a much more delicate investigation. For instance, by applying some general results to be derived in Section 4 to the equation (1), we can prove that under some circumstances mean square and pathwise exponential stability of (1) are transferable from the equation (3) for arbitrary delay constant $h_{1}>0$, but for the time lag $h_{2}>0$ which must be sufficiently small.

One of the main aims of this paper is to give sufficient conditions which contain as a special case the corresponding results in finite dimension (that is, for stochastic ordinary differential equations) to transfer the exponential stability of stochastic partial differential equations to stochastic functional partial differential equations. The problem we are referring to is devoted to the consideration of an infinite dimensional version of (1) in which $f$ has the following form:

$$
f(t, x, y)=A(t, x)+f_{1}(t, y),
$$


with the family of (non-linear) operators $A(t, \cdot)$ satisfying some kinds of coercivity conditions (see Section 2) as well as $f_{1}$ satisfying Lipschitz continuous ones. We would also like to mention that, in some sense, a suitable coercivity condition implies the (exponential) stability of solutions in mean square (and also pathwise exponential stability) in nondelay cases (see Caraballo and Liu [3] and Chow [4]). In addition to this, we will be able to assure exponential stability in mean square (and, as a consequence, pathwise exponential stability) for a great number of finite dimensional stochastic functional differential equations while the results of Mao and Shah [13] only give this kind of stability for certain delay systems in which the delay must be constant and sufficiently small.

Here, we shall analyze only the second moment of solutions. Although we should emphasize that this study can be extended to the $p$-th moment $(p \geq 2)$, which is important if it permits us to obtain some information about the stability of sample paths. We also remark that, as is well known, mean square exponential stability, energy equality and Borel-Cantelli's lemma could imply pathwise exponential stability (see, for instance, Caraballo and Liu [3], Mao [12]).

In Section 2 we begin with some preliminary results. We have not seen a general treatment on existence and uniqueness of strong solutions of stochastic functional differential equations in infinite dimensions in the literature. In Section 3 we shall first establish a result, which is easy to verify in many situations, of existence and uniqueness of strong solutions for a class of stochastic partial functional differential equations. The results of exponential stability are studied in Section 5. Finally, two examples are given in Section 6 to illustrate the theory derived in the preceding sections.

\section{PRELIMINARIES}

First of all, we introduce the framework in which our analysis is going to be carried out. Let $V, H, K$ be real, separable Hilbert spaces such that

$$
V \hookrightarrow H \equiv H^{\prime} \hookrightarrow V^{\prime},
$$

where $V^{\prime}$ is the dual of $V$ and the injections are continuous and dense. In particular, we also assume both $V$ and $V^{\prime}$ are uniformly convex. We denote by $\|\cdot\|,|\cdot|$ and $\|\cdot\|_{*}$ the norms in $V, H$ and $V^{\prime}$ respectively; by $\langle\cdot, \cdot\rangle$ the duality product between $V^{\prime}, V$, and by $(\cdot, \cdot)$ the scalar product in $H$.

Let $w(t)$ be a Wiener process defined on a certain complete probability space $(\Omega, \mathcal{F}, P)$ and take values in the separable Hilbert space $K$, with incremental covariance operator $W$. Let $\left(\mathcal{F}_{t}\right)_{t \geq 0}$ be the $\sigma$-algebras generated by $\{w(s), 0 \leq s \leq t\}$, then $w(t)$ is a martingale relative to $\left(\mathcal{F}_{t}\right)_{t \geq 0}$ and we have the following representation of $w(t)$ :

$$
w(t)=\sum_{i=1}^{\infty} \beta_{i}(t) e_{i},
$$

where $\left\{e_{i}\right\}_{i \geq 1}$ is an orthonormal set of eigenvectors of $W, \beta_{i}(t)$ are mutually independent real Wiener processes with incremental covariance $\lambda_{i}>0, W e_{i}=\lambda_{i} e_{i}$ and $\operatorname{tr} W=$ $\sum_{i=1}^{\infty} \lambda_{i}<\infty(\operatorname{tr}$ denotes the trace of an operator, see Pardoux [16]). 
For an operator $G \in \mathcal{L}(K, H)$, the space of all bounded linear operators from $K$ into $H$, we denote by $\|G\|_{2}$ its Hilbert-Schmidt norm, i.e.

$$
\|G\|_{2}^{2}=\operatorname{tr}\left(G W G^{*}\right)
$$

Given $h \geq 0, p \geq 2$ and $T>0$, we denote by $I^{p}(-h, T ; V)$ the space of all $V$-valued processes $(x(t))_{t \in[-h, T]}$ (we will write $x(t)$ for short) measurable (from $[-h, T] \times \Omega$ into $V$ ), and satisfying:

(1). $x(t)$ is $\mathcal{F}_{t}$-measurable almost surely in $t$ (in the sequel, we will write a.e.t.), where we set $\mathcal{F}_{t}=\mathcal{F}_{0}$ for $t \leq 0$;

(2). $E \int_{-h}^{T}\|x(t)\|^{p} d t<+\infty$.

It is not difficult to check that the space $I^{p}(-h, T ; V)$ is a closed subspace of $L^{p}(\Omega \times$ $[-h, T], \mathcal{F} \otimes \mathcal{B}([-h, T]), d P \otimes d t ; V)$, where $\mathcal{B}([-h, T])$ denotes the Borel $\sigma$-algebra on $[-h, T]$. We also write $L^{2}(\Omega ; C(-h, T ; H))$ instead of $L^{2}(\Omega, \mathcal{F}, d P ; C(-h, T ; H))$, where $C(-h, T ; H)$ denotes the space of all continuous functions from $[-h, T]$ into $H$.

Let $C=C([-h, 0], H)$ be the space of all continuous functions from $[-h, 0]$ into $H$ with sup-norm $\|\psi\|_{C}=\sup _{-h \leq s \leq 0}|\psi(s)|, \psi \in C, L_{V}^{p}=L^{p}([-h, 0] ; V)$ and $L_{H}^{p}=$ $L^{p}([-h, 0] ; H)$.

Given a stochastic process $x(t) \in I^{p}(-h, T ; V) \cap L^{2}(\Omega ; C(-h, T ; H))$, we associate with an $L_{V}^{p} \cap C$-valued stochastic process $x_{t}: \Omega \rightarrow L_{V}^{p} \cap C, t \geq 0$, by setting $x_{t}(s)(\omega)=$ $x(t+s)(\omega), s \in[-h, 0]$.

The first purpose of this paper is to establish an existence and uniqueness result for a class of nonlinear stochastic partial functional differential equations of the form

$$
\left\{\begin{aligned}
d x(t) & =\left(A(t, x(t))+f\left(t, x_{t}\right)\right) d t+g\left(t, x_{t}\right) d w(t), \quad t \in[0, T] \\
x(t) & =\psi(t), \quad t \in[-h, 0]
\end{aligned}\right.
$$

where, in general, the operators are assumed to be nonlinear. In fact, we are interested in the case in which $A(t, \cdot): V \rightarrow V^{\prime}$ is a family of nonlinear monotone and coercive operators, $f(t, \cdot): C \rightarrow H$ and $g(t, \cdot): C \rightarrow \mathcal{L}(K, H)$ are Lipschitz continuous. It is worth pointing out that, in many applications, $A$ usually denotes a partial differential operator (linear or nonlinear), while $f$ and $g$ are first order partial differential ones (cf. [3][16][17]). We will first establish the desired results by a variational type of argument, which is similar to that one carried out by Pardoux's [16] for a case without delays, but subject to necessary changes to make our scheme go through when $f(t, \cdot): C \rightarrow H$ and $g(t, \cdot): C \rightarrow \mathcal{L}(K, H)$. Then we will treat the more general case with $f(t, \cdot): L_{V}^{2} \rightarrow H$ and $g(t, \cdot): L_{V}^{2} \rightarrow \mathcal{L}(K, H)$ by using a Galerkin approximation technique. 


\section{EXISTENCE AND UNIQUENESS OF SOLUTIONS}

Let $A(t, \cdot): V \rightarrow V^{\prime}$ be a family of (nonlinear) operators defined a.e.t. and $p \geq 2$. Assume the following hypotheses:

(a.1) Coercivity: $\exists \alpha>0, \lambda, \nu \in \mathbf{R}^{1}$ such that:

$$
-2\langle A(t, x), x\rangle+\lambda|x|^{2}+\nu \geq \alpha\|x\|^{p}, \quad \forall x \in V \text {, a.e.t.; }
$$

(a.2) Monotonicity:

$$
-2\langle A(t, x)-A(t, y), x-y\rangle+\lambda|x-y|^{2} \geq 0, \forall x, y \in V \text {, a.e.t.; }
$$

(a.3) Boundedness: $\exists \gamma>0$ :

$$
\|A(t, x)\|_{*} \leq \gamma\|x\|^{p-1}, \quad \forall x \in V \text {, a.e.t.; }
$$

(a.4) Hemicontinuity:

$$
\theta \in \mathbf{R}^{1} \rightarrow\langle A(t, x+\theta y), z\rangle \in \mathbf{R}^{1} \text { is continuous } \forall x, y, z \in V \text {, a.e.t.; }
$$

(a.5) Measurability:

$$
t \in(0, T) \rightarrow A(t, x) \in V^{\prime} \text { is Lebesgue - measurable } \forall x \in V \text {, a.e.t.. }
$$

Let $f(t, \cdot): L_{H}^{2} \rightarrow H$ be a family of nonlinear operators defined a.e.t., and satisfy:

(f.1) $f(t, 0)=0$;

(f.2) Lipschitz condition: $\exists k_{1}=k_{1}(h)>0$ such that

$$
|f(t, \eta)-f(t, \xi)| \leq k_{1}\|\eta-\xi\|_{C}, \forall \eta, \xi \in C, \text { a.e.t.; }
$$

(f.3) Measurability: $t \in(0, T) \rightarrow f(t, \eta) \in H$ is Lebesgue-measurable, $\forall \eta \in L_{H}^{2}$.

And let $g(t, \cdot): L_{H}^{2} \rightarrow \mathcal{L}(K, H)$ be another nonlinear operator family defined a.e.t. and satisfy:

(g.1) $g(t, 0)=0$;

(g.2) Lipschitz condition: $\exists k_{2}=k_{2}(h)>0$ such that

$$
\|g(t, \eta)-g(t, \xi)\|_{2} \leq k_{2}\|\eta-\xi\|_{C}, \forall \eta, \quad \xi \in C \text {, a.e.t.; }
$$

(g.3) Measurability: $t \in(0, T) \rightarrow g(t, \eta) \in \mathcal{L}(K, H)$ is Lebesgue-measurable $\forall \eta \in$ $L_{H}^{2}$.

Given an initial value

$$
\psi \in I^{p}(-h, 0 ; V) \cap L^{2}(\Omega ; C(-h, 0 ; H)),
$$


the objective in this section is that under the conditions described above, we hopefully find a unique process $x(t) \in I^{p}(-h, T ; V) \cap L^{2}(\Omega ; C(-h, T ; H))$ such that

$$
\left\{\begin{aligned}
x(t) & =\psi(0)+\int_{0}^{t}\left[A(s, x(s))+f\left(s, x_{s}\right)\right] d s \\
& \quad+\int_{0}^{t} g\left(s, x_{s}\right) d w(s), P-\text { a.s., } \quad \forall t \in[0, T], \\
x(t) & =\psi(t), \quad P \text { - a.s., } \forall t \in[-h, 0] .
\end{aligned}\right.
$$

Remark. (1) First, it is worth mentioning that although the results can be proved for $p>1$, the interesting situations in the applications appear when $p \geq 2$. Because of this, we content ourselves with the analysis of the case $p \geq 2$.

(2). We observe that if $x \in L^{2}(0, T ; C)$, then in view of (f.1)-(f.3), $f(x) \in L^{2}(0, T ; H)$ where $f(x)(t)=f\left(t, x_{t}\right)$. Moreover, the mapping $x \in L^{2}(0, T ; C) \mapsto f(x) \in L^{2}(0, T ; H)$ is continuous and so measurable. Since $\eta \in C \mapsto f(t, \eta) \in H$ is continuous a.e.t., it follows that if $x(t), t \in[-h, T]$ is an $H$-valued and $\mathcal{F}_{t}$-adapted stochastic process, so is $f\left(t, x_{t}\right), t \geq 0$. In addition, if $x \in L^{2}(\Omega \times(0, T) ; C)$, then $f(x) \in L^{2}(\Omega \times(0, T) ; H)$. Finally, if $x^{n}$ is a bounded sequence in $L^{2}(\Omega \times(0, T) ; C), f\left(x^{n}\right)$ is bounded in $L^{2}(\Omega \times$ $(0, T) ; H)$ once again.

Similar results are deduced from (g.1)-(g.3) for $g: L^{2}(0, T ; C) \rightarrow L^{2}(0, T ; \mathcal{L}(K, H))$ defined by $g(x)(t)=g\left(t, x_{t}\right)$. These remarks imply that the integrals appearing in $(*)$ are well defined.

(3). In order to avoid unnecessary technicalities in the following stability analysis, we content ourselves with the consideration of Equation $(*)$ instead of a more general one. However, it is worth pointing out that under some similar conditions, it is possible to extend the results derived here to more general stochastic systems involving coefficients such as $f\left(t, x(t), x_{t}\right)$ and $g\left(t, x(t), x_{t}\right)$ as well as to remove Conditions (f.1), (g.1).

\subsection{Uniqueness of solutions}

Now we shall prove that there exists at most one solution of $(*)$. This result will be deduced mainly from (a.2) and Itô's formula.

Theorem 1. Assume the preceding hypotheses hold. Then, there exists at most one solution of $(*)$ in $I^{p}(-h, T ; V) \cap L^{2}(\Omega ; C(-h, T ; H))$.

Proof. Suppose that $x, y \in I^{p}(-h, T ; V) \cap L^{2}(\Omega ; C(-h, T ; H))$ are two solutions of $(*)$. Then, applying Itô's formula to $(*)$ and taking into account (a.2), we obtain

$$
\begin{aligned}
|x(t)-y(t)|^{2}= & 2 \int_{0}^{t}\langle A(s, x(s))-A(s, y(s)), x(s)-y(s)\rangle d s \\
& +2 \int_{0}^{t}\left(f\left(s, x_{s}\right)-f\left(s, y_{s}\right), x(s)-y(s)\right) d s
\end{aligned}
$$




$$
\begin{aligned}
& +2 \int_{0}^{t}\left(x(s)-y(s),\left(g\left(s, x_{s}\right)-g\left(s, y_{s}\right)\right) d w(s)\right) \\
& +\int_{0}^{t}\left\|g\left(s, x_{s}\right)-g\left(s, y_{s}\right)\right\|_{2}^{2} d s . \\
& \leq \lambda \int_{0}^{t}|x(s)-y(s)|^{2} d s \\
& +2 \int_{0}^{t}\left|x(s)-y(s) \| f\left(s, x_{s}\right)-f\left(s, y_{s}\right)\right| d s \\
& +2 \int_{0}^{t}\left(x(s)-y(s),\left(g\left(s, x_{s}\right)-g\left(s, y_{s}\right)\right) d w(s)\right) \\
& +\int_{0}^{t}\left\|g\left(s, x_{s}\right)-g\left(s, y_{s}\right)\right\|_{2}^{2} d s .
\end{aligned}
$$

Now, it follows from (f.2) and (g.2) that for any $t \in[0, T]$

$$
\begin{aligned}
E \sup _{0 \leq s \leq t}|x(s)-y(s)|^{2} \leq & (|\lambda|+1) \int_{0}^{t} E|x(s)-y(s)|^{2} d s+\left(k_{1}^{2}+k_{2}^{2}\right) \int_{0}^{t} E\left\|x_{s}-y_{s}\right\|_{C}^{2} d s \\
& +2 E \sup _{0 \leq s \leq t} \int_{0}^{s}\left(x(r)-y(r),\left(g\left(r, x_{r}\right)-g\left(r, y_{r}\right)\right) d w(r)\right) .
\end{aligned}
$$

However, by Burkholder-Davis-Gundy's inequality, we have

$$
\begin{aligned}
E \sup _{0 \leq s \leq t} & \int_{0}^{s}\left(x(r)-y(r),\left(g\left(r, x_{r}\right)-g\left(r, y_{r}\right)\right) d w(r)\right) \\
& \leq 3 E\left\{\sup _{0 \leq s \leq t}|x(s)-y(s)|\left[\int_{0}^{t}\left\|g\left(s, x_{s}\right)-g\left(s, y_{s}\right)\right\|_{2}^{2} d s\right]^{1 / 2}\right\} \\
& \leq \frac{1}{4} E \sup _{0 \leq s \leq t}|x(s)-y(s)|^{2}+K \int_{0}^{t} E\left\|g\left(s, x_{s}\right)-g\left(s, y_{s}\right)\right\|_{2}^{2} d s \\
& \leq \frac{1}{4} E \sup _{0 \leq s \leq t}|x(s)-y(s)|^{2}+K \cdot k_{2}^{2} \int_{0}^{t} E\left\|x_{s}-y_{s}\right\|_{C}^{2} d s
\end{aligned}
$$

for some positive constant $K>0$. On the other hand, since $x(s)=y(s)$ for $s \leq 0$, we easily get

$$
\begin{aligned}
\int_{0}^{t} E\left\|x_{s}-y_{s}\right\|_{C}^{2} d s & =\int_{0}^{t} E \sup _{-h \leq r \leq 0}\left|x_{s}(r)-y_{s}(r)\right|^{2} d s \\
& =\int_{0}^{t} E \sup _{-h \leq r \leq 0}|x(s+r)-y(s+r)|^{2} d s \\
& \leq \int_{0}^{t} E \sup _{0 \leq r \leq s}|x(r)-y(r)|^{2} d s .
\end{aligned}
$$


Thus, it follows from (7)-(9)

$E \sup _{0 \leq s \leq t}|x(s)-y(s)|^{2} \leq 2\left[|\lambda|+1+k_{1}^{2}+k_{2}^{2}+2 k_{2}^{2} K\right] \int_{0}^{t} E \sup _{0 \leq r \leq s}|x(r)-y(r)|^{2} d s, \quad \forall t \in[0, T]$.

Now, Gronwall's lemma obviously implies uniqueness.

Remark. (1) Observe that if we assume the following monotonicity hypothesis

(a.2)' For all $\xi, \eta \in L^{p}(-h, T ; V)$ with $\xi_{0}=\eta_{0}$ such that

$$
\begin{aligned}
-2\langle A(t, \xi(t)) & \left.+f\left(t, \xi_{t}\right)-A(t, \eta(t))-f\left(t, \eta_{t}\right), \xi(t)-\eta(t)\right\rangle+\lambda|\xi(t)-\eta(t)|^{2} \\
& \geq\left\|g\left(t, \xi_{t}\right)-g\left(t, \eta_{t}\right)\right\|_{2}^{2} \quad \text { a.e.t } \in[0, T]
\end{aligned}
$$

instead of (a.2), uniqueness would have been easily deduced. Indeed, notice that in this case, Itô's formula and (a.2)' imply

$$
E|x(t)-y(t)|^{2} \leq \lambda \int_{0}^{t} E|x(s)-y(s)|^{2} d s \quad \forall t \in[0, T]
$$

for arbitrary two solutions $x, y$ of the problem. Moreover, it is sufficient to assume an integral version of (a.2)', namely,

(a.2)" For all $\xi, \eta \in L^{p}(-h, T ; V)$ with $\xi_{0}=\eta_{0}$ such that

$$
\begin{aligned}
-2 \int_{0}^{t}\langle A(s, \xi(s))+ & \left.f\left(s, \xi_{s}\right)-A(s, \eta(s))-f\left(s, \eta_{s}\right), \xi(s)-\eta(s)\right\rangle d s \\
& +\lambda \int_{0}^{t}|\xi(s)-\eta(s)|^{2} d s \geq \int_{0}^{t}\left\|g\left(s, \xi_{s}\right)-g\left(s, \eta_{s}\right)\right\|_{2}^{2} d s \quad \text { a.e.t } \in[0, T] .
\end{aligned}
$$

(2) Conversely, it is not difficult to prove by carrying out similar computations to the ones in (8) that (a.2), (f.2) and (g.2) imply (a.2)" (of course, with different parameter $\lambda$ from that one in (a.2)).

\subsection{Existence of strong solutions}

First of all, we state a theorem on existence and uniqueness of solutions of stochastic evolution equations. Next, by means of this result we will prove the desired existence of solution of $(*)$.

Theorem 2. Assume (a.1)-(a.5) hold with $\lambda=0$. Then, there exists a unique process $x \in I^{p}(0, T ; V) \cap L^{2}(\Omega ; C(0, T ; H))$ such that

$$
x(t)=\psi(0)+\int_{0}^{t}\left[A(s, x(s))+f_{1}(s)\right] d s+M(t), P-\text { a.s. }, \quad \forall t \in[0, T],
$$


where $f_{1} \in I^{2}(0, T ; H), \psi(0) \in L^{2}\left(\Omega, \mathcal{F}_{0}, P ; H\right)$ and $M(t)$ is an $H$-valued continuous, square integrable $\mathcal{F}_{t}$-martingale. In addition, the following energy equality also holds:

$$
\begin{aligned}
|x(t)|^{2}= & |\psi(0)|^{2}+2 \int_{0}^{t}\langle A(s, x(s)), x(s)\rangle d s \\
& +2 \int_{0}^{t}\left(f_{1}(s), x(s)\right) d s+2 \int_{0}^{t}(x(s), d M(s))+\operatorname{tr}\langle\langle M\rangle\rangle_{t}, \quad P-\text { a.s. }, \quad \forall t \in[0, T],
\end{aligned}
$$

where $\langle\langle M\rangle\rangle_{t}$ denotes the quadratic variation of $M(t)$.

Proof. See Métivier and Pellaumail [14].

Now we are in a position to prove the existence of solution to the problem $(*)$.

Theorem 3. Assume that (a.1)-(a.5), (f.1)-(f.3) and (g.1)-(g.3) hold. Then, for each $\psi \in I^{p}(-h, 0 ; V) \cap L^{2}(\Omega ; C(-h, 0 ; H))$ there exists a unique solution of the problem $(*)$ in $I^{p}(-h, T ; V) \cap L^{2}(\Omega ; C(-h, T ; H))$.

Proof. Uniqueness holds from Theorem 1.

For the existence, we consider the equations

$$
\begin{gathered}
x^{1}(t)=\psi(0)+\int_{0}^{t}\left[A\left(s, x^{1}(s)\right)-\frac{\lambda}{2} x^{1}(s)\right] d s, t \in[0, T], \\
x^{1}(t)=\psi(t), \quad t \in[-h, 0], \\
x^{n+1}(t)=\psi(0)+\int_{0}^{t}\left[A\left(s, x^{n+1}(s)\right)-\frac{\lambda}{2} x^{n+1}(s)\right] d s+\int_{0}^{t} \frac{\lambda}{2} x^{n}(s) d s \\
+\int_{0}^{t} f\left(s, x_{s}^{n}\right) d s+\int_{0}^{t} g\left(s, x_{s}^{n}\right) d w(s), \quad t \in[0, T], \quad \forall n \geq 1 \\
x^{n+1}(t)=\psi(t), t \in[-h, 0], \quad \forall n \geq 1 .
\end{gathered}
$$

By virtue of (a.1)-(a.5), the family $A_{1}(t,):. V \rightarrow V^{\prime}$ defined as $A_{1}(t, x)=A(t, x)-$ $(\lambda / 2) x$, satisfies the assumptions in Theorem 2. Consequently, (10)-(12) has a unique solution $x^{1} \in I^{p}(-h, T ; V) \cap L^{2}(\Omega ; C(-h, T ; H))$.

We note that, from (f.2) and (g.2) it follows:

i) The mapping $(t, \omega) \in(0, T) \times \Omega \mapsto f\left(t, x_{t}^{1}\right) \in H$ belongs to $I^{2}(0, T ; H)$;

ii) The mapping $(t, \omega) \in(0, T) \times \Omega \mapsto g\left(t, x_{t}^{1}\right) \in \mathcal{L}(K, H)$ belongs to the space $I^{2}(0, T ; \mathcal{L}(K, H))$ and therefore $\int_{0}^{\cdot} g\left(t, x_{s}^{1}\right) d w(s)$ is a continuous and square integrable $\mathcal{F}_{t}$-martingale.

Consequently, bearing these remarks in mind we can use Theorem 2 and get that there exists a unique process $x^{2} \in I^{p}(-h, T ; V) \cap L^{2}(\Omega ; C(-h, T ; H))$, which is the solution of (11)-(12) for $n=1$. By recurrence, we obtain a sequence of solutions for (10)-(12), $\left\{x^{n}\right\}_{n \geq 1} \subset I^{p}(-h, T ; V) \cap L^{2}(\Omega ; C(-h, T ; H))$. 
Now, we want to prove that the sequence $\left\{x^{n}\right\}$ is convergent to a process $x$ in $I^{p}(-h, T ; V) \cap L^{2}(\Omega ; C(-h, T ; H))$, which will be the solution of $(*)$. For this end, we shall first prove the following lemmas.

Lemma 1. $\left\{x^{n}\right\}$ is a Cauchy sequence in $L^{2}(\Omega ; C(-h, T ; H))$.

Proof. Indeed, for $n>1$ and the process $x^{n+1}(t)-x^{n}(t)$, it follows from Itô's formula

$$
\begin{aligned}
\left|x^{n+1}(t)-x^{n}(t)\right|^{2}= & 2 \int_{0}^{t}\left\langle A\left(x^{n+1}\right)-A\left(x^{n}\right), x^{n+1}-x^{n}\right\rangle d s \\
& -\lambda \int_{0}^{t}\left|x^{n+1}-x^{n}\right|^{2} d s+\lambda \int_{0}^{t}\left(x^{n+1}-x^{n}, x^{n}-x^{n-1}\right) d s \\
& +2 \int_{0}^{t}\left(f\left(x^{n}\right)-f\left(x^{n-1}\right), x^{n+1}-x^{n}\right) d s \\
& +2 \int_{0}^{t}\left(x^{n+1}-x^{n},\left(g\left(x^{n}\right)-g\left(x^{n-1}\right)\right) d w(s)\right) \\
& +\int_{0}^{t}\left\|g\left(x^{n}\right)-g\left(x^{n-1}\right)\right\|_{2}^{2} d s
\end{aligned}
$$

where, by definition, $x^{n}:=x^{n}(s), \quad A\left(x^{n}\right):=A\left(s, x^{n}(s)\right), \quad f\left(x^{n}\right):=f\left(s, x_{s}^{n}\right)$ and $g\left(x^{n}\right):=g\left(s, x_{s}^{n}\right)$ ¿From (a.2), it is easy to deduce

$$
\begin{aligned}
\left|x^{n+1}(t)-x^{n}(t)\right|^{2} \leq & |\lambda| \int_{0}^{t}\left|x^{n+1}-x^{n}\right|\left|x^{n}-x^{n-1}\right| d s \\
& +2 \int_{0}^{t}\left|f\left(x^{n}\right)-f\left(x^{n-1}\right)\right|\left|x^{n+1}-x^{n}\right| d s \\
& +2\left|\int_{0}^{t}\left(x^{n+1}-x^{n},\left(g\left(x^{n}\right)-g\left(x^{n-1}\right)\right) d w(s)\right)\right| \\
& +\int_{0}^{t}\left\|g\left(x^{n}\right)-g\left(x^{n-1}\right)\right\|_{2}^{2} d s .
\end{aligned}
$$

Consequently, (14) yields

$$
\begin{aligned}
E\left[\sup _{0 \leq \theta \leq t} \mid\right. & \left.x^{n+1}(\theta)-\left.x^{n}(\theta)\right|^{2}\right] \\
\leq & |\lambda| E \int_{0}^{t}\left|x^{n+1}-x^{n}\right|\left|x^{n}-x^{n-1}\right| d s \\
& +2 E \int_{0}^{t}\left|f\left(x^{n}\right)-f\left(x^{n-1}\right)\right|\left|x^{n+1}-x^{n}\right| d s \\
& +2 E\left[\sup _{0 \leq \theta \leq t}\left|\int_{0}^{\theta}\left(x^{n+1}-x^{n},\left(g\left(x^{n}\right)-g\left(x^{n-1}\right)\right) d w(s)\right)\right|\right] \\
& +E \int_{0}^{t}\left\|g\left(x^{n}\right)-g\left(x^{n-1}\right)\right\|_{2}^{2} d s .
\end{aligned}
$$


Now, we estimate the terms on the right-hand side of (15) by using the inequality

$$
2 a b \leq \frac{a^{2}}{l^{2}}+l^{2} b^{2}, a, b \in \mathbf{R}^{1}, l>0,
$$

for an appropriate $l>0$. Firstly, it can be deduced

$$
\begin{aligned}
|\lambda| E \int_{0}^{t}\left|x^{n+1}-x^{n}\right|\left|x^{n}-x^{n-1}\right| d s \leq & \frac{1}{4} E\left[\sup _{0 \leq \theta \leq t}\left|x^{n+1}(\theta)-x^{n}(\theta)\right|^{2}\right] \\
& +\lambda^{2} T \int_{0}^{t} E\left[\sup _{0 \leq \theta \leq s}\left|x^{n}(\theta)-x^{n-1}(\theta)\right|^{2}\right] d s .
\end{aligned}
$$

On the other hand, since $x^{n}(s)=x^{n-1}(s),-h \leq s \leq 0$, we can get from (g.2)

$$
\begin{aligned}
E \int_{0}^{t}\left\|g\left(x^{n}\right)-g\left(x^{n-1}\right)\right\|_{2}^{2} d s & \leq k_{2}^{2} E \int_{0}^{t}\left\|x_{s}^{n}-x_{s}^{n-1}\right\|_{C}^{2} d s \\
& =k_{2}^{2} E \int_{0}^{t} \sup _{-h \leq r \leq 0}\left|x_{s}^{n}(r)-x_{s}^{n-1}(r)\right|^{2} d s \\
& \leq k_{2}^{2} E \int_{0}^{t} \sup _{0 \leq r \leq s}\left|x^{n}(r)-x^{n-1}(r)\right|^{2} d s,
\end{aligned}
$$

and, in a similar manner, from (f.2) we can obtain

$$
\begin{aligned}
2 E \int_{0}^{t}\left|f\left(x^{n}\right)-f\left(x^{n-1}\right)\right|\left|x^{n+1}-x^{n}\right| d s \leq & \frac{1}{4 T} E \int_{0}^{t}\left|x^{n+1}-x^{n}\right|^{2} d s \\
& +4 k_{1}^{2} T E \int_{0}^{t}\left\|x_{s}^{n}-x_{s}^{n-1}\right\|_{C}^{2} d s \\
\leq & \frac{1}{4} E\left[\sup _{0 \leq r \leq t}\left|x^{n+1}(r)-x^{n}(r)\right|^{2}\right] \\
& +4 k_{1}^{2} T \int_{0}^{t} E\left[\sup _{0 \leq r \leq s}\left|x^{n}(r)-x^{n-1}(r)\right|^{2}\right] d s .
\end{aligned}
$$

Now, Burkholder-Davis-Gundy's inequality implies

$$
\begin{aligned}
2 E\left[\sup _{0 \leq r \leq t} \mid\right. & \left.\int_{0}^{r}\left(x^{n+1}-x^{n},\left(g\left(x^{n}\right)-g\left(x^{n-1}\right)\right) d w(s)\right) \mid\right] \\
\leq & 6 E\left[\left(\sup _{0 \leq r \leq t}\left|x^{n+1}(r)-x^{n}(r)\right|^{2}\right) \int_{0}^{t}\left\|g\left(x^{n}\right)-g\left(x^{n-1}\right)\right\|_{2}^{2} d s\right]^{1 / 2} \\
\leq & \frac{1}{4} E\left[\sup _{0 \leq r \leq t}\left|x^{n+1}(r)-x^{n}(r)\right|^{2}\right] \\
& +72 k_{2}^{2} \int_{0}^{t} E\left[\sup _{0 \leq r \leq s}\left|x^{n}(r)-x^{n-1}(r)\right|^{2}\right] d s .
\end{aligned}
$$


If we set

$$
\varphi^{n}(t)=E\left[\sup _{0 \leq \theta \leq t}\left|x^{n+1}(\theta)-x^{n}(\theta)\right|^{2}\right]
$$

then from (15)-(19), it could be deduced that there exists a positive constant $c>0$ such that

$$
\varphi^{n}(t) \leq \frac{3}{4} \varphi^{n}(t)+c \int_{0}^{t} \varphi^{n-1}(s) d s
$$

and consequently there exists $k>0$ such that

$$
\varphi^{n}(t) \leq k \int_{0}^{t} \varphi^{n-1}(s) d s
$$

By iteration from (22), we get

$$
\varphi^{n}(t) \leq \frac{k^{n-1} T^{n-1}}{(n-1) !} \varphi^{1}(T), \forall n>1, \quad \forall t \in[0, T] .
$$

Therefore,

$$
E\left[\sup _{0 \leq \theta \leq T}\left|x^{n+1}(\theta)-x^{n}(\theta)\right|^{2}\right] \leq \frac{k^{n-1} T^{n-1}}{(n-1) !} \varphi^{1}(T), \quad \forall n>1 .
$$

Obviously, since $x^{n+1}(\theta)=x^{n}(\theta)$ for $\theta \in[-h, 0]$, (24) implies that $\left\{x^{n}\right\}$ is a Cauchy sequence in $L^{2}(\Omega ; C(-h, T ; H))$.

Lemma 2. The sequence $\left\{x^{n}\right\}$ is bounded in $I^{p}(-h, T ; V)$.

Proof. Indeed, applying Itô's formula to $\left|x^{n}\right|^{2}$ with $n \geq 2$ immediately yields

$$
\begin{aligned}
E\left|x^{n}(T)\right|^{2}= & 2 E \int_{0}^{T}\left\langle A\left(x^{n}\right), x^{n}\right\rangle d s-\lambda E \int_{0}^{T}\left|x^{n}\right|^{2} d s \\
& +E|\psi(0)|^{2}+2 E \int_{0}^{T}\left(f\left(x^{n-1}\right), x^{n}\right) d s \\
& +\lambda E \int_{0}^{T}\left(x^{n}, x^{n-1}\right) d s+E \int_{0}^{T}\left\|g\left(x^{n-1}\right)\right\|_{2}^{2} d s .
\end{aligned}
$$

Therefore,

$$
\begin{aligned}
-2 E \int_{0}^{T} & \left\langle A\left(x^{n}\right), x^{n}\right\rangle d s+\lambda E \int_{0}^{T}\left|x^{n}\right|^{2} d s \\
\leq & E|\psi(0)|^{2}+2 E \int_{0}^{T}\left|f\left(x^{n-1}\right)\right|\left|x^{n}\right| d s \\
& +|\lambda| E \int_{0}^{T}\left|x^{n}\left\|x^{n-1} \mid d s+E \int_{0}^{T}\right\| g\left(x^{n-1}\right) \|_{2}^{2} d s .\right.
\end{aligned}
$$

Since $\left\{x^{n}\right\}$ is convergent in $L^{2}(\Omega ; C(-h, T ; H))$, it will be bounded in this space. Now, it is not difficult to check that there exists a positive constant $k^{\prime}>0$ such that the right-hand 
side of (26) is bounded by this constant. For instance, we will estimate one of those terms. Firstly, we observe that

$$
\begin{aligned}
\int_{0}^{T}\left\|x_{s}^{n-1}\right\|_{C}^{2} d s & =\int_{0}^{T} \sup _{-h \leq r \leq 0}\left|x^{n-1}(s+r)\right|^{2} d s \\
& \leq \int_{0}^{T} \sup _{-h \leq \theta \leq s}\left|x^{n-1}(\theta)\right|^{2} d s .
\end{aligned}
$$

Next,

$$
\begin{aligned}
2 E \int_{0}^{T}\left|f\left(x^{n-1}\right) \| x^{n}\right| d s & \leq 2 k_{1} E \int_{0}^{T}\left\|x_{s}^{n-1}\right\|_{C}\left|x^{n}(s)\right| d s \\
& \leq k_{1} E \int_{0}^{T}\left[\left\|x_{s}^{n-1}\right\|_{C}^{2}+\left|x^{n}(s)\right|^{2}\right] d s \\
& \leq T k_{1} E\left(\sup _{-h \leq \theta \leq T}\left|x^{n-1}(\theta)\right|^{2}\right)+k_{1} T E\left(\sup _{0 \leq \theta \leq T}\left|x^{n}(\theta)\right|^{2}\right) \\
& =T k_{1}\left\|x^{n-1}\right\|_{L^{2}(\Omega ; C(-h, T ; H))}^{2}+k_{1} T\left\|x^{n}\right\|_{L^{2}(\Omega ; C(0, T ; H))}^{2},
\end{aligned}
$$

which, in addition to (26) and (a.1), leads to the following inequalities:

$$
\alpha \int_{0}^{T} E\left\|x^{n}(s)\right\|^{p} d s \leq-2 E \int_{0}^{T}\left\langle A\left(x^{n}\right), x^{n}\right\rangle d s+\lambda E \int_{0}^{T}\left|x^{n}\right|^{2} d s+\nu T \leq k^{\prime},
$$

and Lemma 2 is proved.

Lemma 3. The limit of the sequence $\left\{x^{n}\right\}$ is a solution to $(*)$.

Proof. Firstly, we observe that Lemma 1 implies that there exists $x \in L^{2}(\Omega ; C(-h, T ; H))$ such that $x^{n} \rightarrow x$ in $L^{2}(\Omega ; C(-h, T ; H))$. Now, since (f.2) and (g.2) hold, we have $f\left(x^{n}\right) \rightarrow f(x)\left(\right.$ in $\left.L^{2}\left(\Omega ; L^{\infty}(0, T ; H)\right)\right)$, and $g\left(x^{n}\right) \rightarrow g(x)\left(\right.$ in $\left.L^{2}\left(\Omega ; L^{\infty}(0, T ; \mathcal{L}(K, H))\right)\right)$.

On the other hand, by virtue of Lemma $2\left\{x^{n}\right\}$ has a subsequence which is weakly convergent in $I^{p}(-h, T ; V)$. But, since $x^{n} \rightarrow x$ in $L^{2}(\Omega ; C(-h, T ; H))$, we can assure that $x^{n} \rightarrow x$ weakly in $I^{p}(-h, T ; V)$ (in the sequel, we will denote this by $x^{n} \rightarrow x$ in $\left.I^{p}(-h, T ; V)\right)$. Nevertheless, it follows from (a.3) that $\left\{A\left(x^{n}\right)\right\}$ is bounded in $L^{p^{\prime}}(\Omega \times$ $\left.(0, T) ; V^{\prime}\right)\left(\right.$ with $p^{\prime}$ such that $\left.(1 / p)+\left(1 / p^{\prime}\right)=1\right)$, since

$$
\int_{0}^{T} E\left\|A\left(t, x^{n}(t)\right)\right\|_{*}^{p /(p-1)} d t \leq \gamma \int_{0}^{T} E\left\|x^{n}(t)\right\|^{p} d t \leq \gamma k^{\prime} / \alpha
$$

Therefore, from each subsequence of $\left\{A\left(x^{n}\right)\right\}$, we can get another subsequence weakly convergent in $L^{p^{\prime}}\left(\Omega \times(0, T) ; V^{\prime}\right)$. Now, we will see that all the limits of different subsequences coincide. Indeed, let $v_{1}, v_{2}$ be the limits of two different subsequences. Since $x^{n} \rightarrow x$ in $L^{2}(\Omega ; C(-h, T ; H)), f\left(x^{n}\right) \rightarrow f(x)$ in $L^{2}\left(\Omega ; L^{\infty}(0, T ; H)\right)$ and $g\left(x^{n}\right) \rightarrow g(x)$ 
in $L^{2}\left(\Omega ; L^{\infty}(0, T ; \mathcal{L}(K, H))\right)$, then (11) implies that the whole sequence $\int_{t_{1}}^{t_{2}} A\left(s, x^{n}(s)\right) d s$ converges in $L^{1}\left(\Omega ; V^{\prime}\right)$ for all $t_{1}, t_{2} \in[0, T]$, and hence

$$
\left.\int_{t_{1}}^{t_{2}} v_{1}(s) d s=\int_{t_{1}}^{t_{2}} v_{2}(s) d s \forall t_{1}, t_{2} \in[0, T] \quad \text { (equality in } L^{p^{\prime}}\left(\Omega ; V^{\prime}\right)\right) .
$$

¿From this, it follows that $v_{1}=v_{2}$ in $L^{p^{\prime}}\left(\Omega \times(0, T) ; V^{\prime}\right)$ and finally $A\left(x^{n}\right) \rightarrow v$ in $L^{p^{\prime}}\left(\Omega \times(0, T) ; V^{\prime}\right)$. In conclusion, we have proved:

$$
\begin{gathered}
x^{n} \rightarrow x \text { in } L^{2}(\Omega ; C(0, T ; H)), \\
f\left(x^{n}\right) \rightarrow f(x) \text { in } L^{2}\left(\Omega ; L^{\infty}(0, T ; H)\right), \\
g\left(x^{n}\right) \rightarrow g(x) \text { in } L^{2}\left(\Omega ; L^{\infty}(0, T ; \mathcal{L}(K, H))\right), \\
x^{n} \rightarrow x \text { in } I^{p}(-h, T ; V), \\
A\left(x^{n}\right) \rightarrow v \text { in } L^{p^{\prime}}\left(\Omega \times(0, T) ; V^{\prime}\right) .
\end{gathered}
$$

Finally, since (27)-(31) hold, we can take limits in (11) and obtain

$$
x(t)=\psi(0)+\int_{0}^{t} v(s) d s+\int_{0}^{t} f\left(s, x_{s}\right) d s+\int_{0}^{t} g\left(s, x_{s}\right) d w_{s} .
$$

Thus, in order to finish the proof of the theorem, it is sufficient to prove that $A(s, x(s))=$ $v(s)$ in $L^{p^{\prime}}\left(\Omega \times(0, T) ; V^{\prime}\right)$. However, from (25) it is easy to deduce

$$
\begin{aligned}
2 E \int_{0}^{T}\left\langle A\left(x^{n}\right), x^{n}\right\rangle d s= & \lambda E \int_{0}^{T}\left|x^{n}\right|^{2} d s+E\left|x^{n}(T)\right|^{2}-E|\psi(0)|^{2} \\
& -2 E \int_{0}^{T}\left(f\left(x^{n-1}\right), x^{n}\right) d s-\lambda E \int_{0}^{T}\left(x^{n}, x^{n-1}\right) d s \\
& -E \int_{0}^{T}\left\|g\left(x^{n-1}\right)\right\|_{2}^{2} d s,
\end{aligned}
$$

which, together with (27)-(29), immediately implies

$$
\begin{aligned}
\lim _{n \rightarrow \infty} 2 E \int_{0}^{T}\left\langle A\left(x^{n}\right), x^{n}\right\rangle d s= & E|x(T)|^{2}-E|\psi(0)|^{2} \\
& -2 E \int_{0}^{T}(f(x), x) d s-E \int_{0}^{T}\|g(x)\|_{2}^{2} d s .
\end{aligned}
$$

However, (32) and Itô's formula yield

$$
\lim _{n \rightarrow \infty} E \int_{0}^{T}\left\langle A\left(x^{n}\right), x^{n}\right\rangle d s=E \int_{0}^{T}\langle v, x\rangle d s .
$$


By virtue of (a.2), we get

$$
-2 E \int_{0}^{T}\left\langle A\left(x^{n}\right)-A(z), x^{n}-z\right\rangle d s+\lambda E \int_{0}^{T}\left|x^{n}-z\right|^{2} d s \geq 0
$$

for all $z \in L^{p}(\Omega \times(0, T) ; V) \cap L^{2}(\Omega \times(0, T) ; H)$. Nevertheless, (27), (30) and (31) allow us to take limits in (36) and, it follows

$$
-2 E \int_{0}^{T}\langle v-A(z), x-z\rangle d s+\lambda E \int_{0}^{T}|x-z|^{2} d s \geq 0 .
$$

Now, if we set $z=x-\theta z_{2}\left(\right.$ for $\left.\theta>0, z_{2} \in L^{p}(\Omega \times(0, T) ; V) \cap L^{2}(\Omega \times(0, T) ; H)\right)$, we get

$$
-2 E \int_{0}^{T}\left\langle v-A\left(x-\theta z_{2}\right), \theta z_{2}\right\rangle d s+\lambda \theta^{2} E \int_{0}^{T}\left|z_{2}\right|^{2} d s \geq 0 .
$$

In (38), we divide by $\theta$, take limit as $\theta \rightarrow 0$ and then use the hemicontinuity (a.4) to obtain:

$$
-E \int_{0}^{T}\left\langle v-A(x), z_{2}\right\rangle d s \geq 0, \forall z_{2} \in L^{p}(\Omega \times(0, T) ; V) \cap L^{2}(\Omega \times(0, T) ; H),
$$

and therefore $v=A(x)$. Since (32) is true with $v=A(x)$, the proof of Theorem 3 is now complete.

\section{EXISTENCE AND UNIQUENESS BY A GALERKIN APPROXIMATION}

First of all, we would like to point out that in many situations, it is convenient to consider another norm $\|\cdot\|_{L_{H}^{2}}$ instead of $\|\cdot\|_{C}$ for initial datum spaces in $(*)$. The arguments in the last section still carry through when the norm $\|\cdot\|_{C}$ is replaced by $\|\cdot\|_{L_{H}^{2}}$ in (f.2) and (g.2). In this section we shall investigate an existence and uniqueness result for (*) in a

more general situation. Precisely, let us assume hypotheses (a.1)-(a.5) for the family of operators $A(t, \cdot)$. Suppose $f(t, \cdot): L_{H}^{2} \rightarrow H$ is a family of nonlinear operators defined a.e.t. and satisfying:

(F.1) $f(t, 0)=0$;

(F.2) Lipschitz condition: $\exists k_{1}=k_{1}(h)>0$ such that

$$
|f(t, \eta)-f(t, \xi)| \leq k_{1}\|\eta-\xi\|_{L_{V}^{2}}, \forall \eta, \xi \in L_{V}^{2} \text {, a.e.t.; }
$$

(F.3) Measurability: $t \in(0, T) \rightarrow f(t, \eta) \in H$ is Lebesgue-measurable, $\forall \eta \in L_{V}^{2}$.

And let $g(t, \cdot): L_{H}^{2} \rightarrow \mathcal{L}(K, H)$ be another nonlinear operator family defined a.e.t. and satisfying: 
(G.1) $g(t, 0)=0$;

(G.2) Lipschitz condition: $\exists k_{2}=k_{2}(h)>0$ such that

$$
\|g(t, \eta)-g(t, \xi)\|_{2} \leq k_{2}\|\eta-\xi\|_{L_{V}^{2}}, \forall \eta, \xi \in L_{V}^{2} \text {, a.e.t.; }
$$

(G.3) Measurability: $t \in(0, T) \rightarrow g(t, \eta) \in \mathcal{L}(K, H)$ is Lebesgue-measurable $\forall \eta \in$ $L_{V}^{2}$.

Theorem 4. In addition to (a.1)-(a.5), (F.1)-(F.3) and (G.1)-(G.3), suppose the two following hypotheses hold:

(C) There exist $\alpha>0, \lambda, \nu, \tau \in \mathbf{R}^{1}$ such that for all $\xi \in L^{p}(-h, T ; V)$

$$
\begin{array}{r}
-2\left\langle A(t, \xi(t))+f\left(t, \xi_{t}\right), \xi(t)\right\rangle+\lambda|\xi(t)|^{2}+\tau\left\|\xi_{0}\right\|_{L_{V}^{p}}^{2}+\nu \\
\geq \alpha\|\xi(t)\|^{p}+\left\|g\left(t, \xi_{t}\right)\right\|_{2}^{2} \quad \text { a.e.t } \in[0, T]
\end{array}
$$

(M) For all $\xi, \eta \in L^{p}(-h, T ; V)$ with $\xi_{0}=\eta_{0}$,

$$
\begin{aligned}
-2\left\langle A(t, \xi(t))+f\left(t, \xi_{t}\right)\right. & \left.-A(t, \eta(t))-f\left(t, \eta_{t}\right), \xi(t)-\eta(t)\right\rangle+\lambda|\xi(t)-\eta(t)|^{2} \\
& \geq\left\|g\left(t, \xi_{t}\right)-g\left(t, \eta_{t}\right)\right\|_{2}^{2} \quad \text { a.e.t } \in[0, T] .
\end{aligned}
$$

Then, for each $\psi \in I^{p}(-h, 0 ; V) \cap L^{2}(\Omega ; C(-h, 0 ; H))$ there exists a unique solution of the problem $(*)$ in $I^{p}(-h, T ; V) \cap L^{2}(\Omega ; C(-h, T ; H))$.

Proof. Uniqueness follows immediately from Itô's formula, Assumption (M) and Gronwall's lemma. Indeed, let $x, y \in I^{p}(-h, T ; V) \cap L^{2}(\Omega ; C(-h, T ; H))$ be two solutions to (*). Then, it is easy to obtain

$$
\begin{aligned}
E|x(t)-y(t)|^{2}= & 2 \int_{0}^{t} E\langle A(s, x(s))-A(s, y(s)), x(s)-y(s)\rangle d s \\
& +2 \int_{0}^{t} E\left(f\left(s, x_{s}\right)-f\left(s, y_{s}\right), x(s)-y(s)\right) d s \\
& +\int_{0}^{t} E\left\|g\left(s, x_{s}\right)-g\left(s, y_{s}\right)\right\|_{2}^{2} d s . \\
\leq & \lambda \int_{0}^{t} E|x(s)-y(s)|^{2} d s
\end{aligned}
$$

from which uniqueness follows by means of Gronwall's lemma.

As for the existence, we shall split the proof into the following four steps.

STEP 1. Finite-dimensional approximation

Let $\left\{v_{1}, v_{2}, \ldots, v_{n}, \ldots\right\}$ be an orthonormal basis of $H$ where $v_{i} \in V$ for all $i \geq 1$. Let $V_{n}=H_{n}=V_{n}^{\prime}$ denote the vector space generated by $\left\{v_{1}, \ldots v_{n}\right\}$. Let $P_{n} \in \mathcal{L}\left(H, H_{n}\right)$ be 
the orthogonal projection from $H$ onto $H_{n}$. Then, $P_{n}$ can be extended to an operator $\tilde{P}_{n}$ from $V^{\prime}$ onto $V_{n}^{\prime}$ in the following way

$$
\tilde{P}_{n} u=\sum_{i=1}^{n}\left\langle u, v_{i}\right\rangle v_{i}, u \in V^{\prime} .
$$

Let $\left\{l_{1}, l_{2}, \ldots, l_{n}, \ldots\right\}$ denote an orthonormal basis in $K$, and let $\pi_{n} \in \mathcal{L}\left(K, K_{n}\right)$ be the projection from $K$ onto $K_{n}=\operatorname{span}\left\{l_{1}, \ldots, l_{n}\right\}$.

Now we consider the problem

$$
\left\{\begin{array}{c}
d\left(x^{n}(t), v_{i}\right)=\left\langle A\left(t, x^{n}(t)\right)+f\left(t, x_{t}^{n}\right), v_{i}\right\rangle d t \\
\quad+\left(v_{i}, g\left(t, x_{t}^{n}\right) d\left(\pi_{n} w(t)\right)\right), \quad 1 \leq i \leq n, \\
x^{n}(t)=P_{n} \psi(t), \quad t \in[-h, 0] .
\end{array}\right.
$$

This equation can be rewritten in an equivalent way as follows. Let $A^{n}(t, \cdot)$ denote the family of operators from $V_{n}$ into $V_{n}^{\prime}$ defined as $A^{n}(t, x)=\tilde{P}_{n} A(t, x), x \in V_{n}$. Assume $f^{n}(t, \cdot): L_{H_{n}}^{2} \rightarrow H_{n}$ given by $f^{n}(t, \xi)=P_{n} f(t, \xi)$ for $\xi \in L_{H_{n}}^{2}, g^{n}(t, \cdot): L_{H_{n}}^{2} \rightarrow \mathcal{L}\left(K_{n}, H_{n}\right)$ defined by $g^{n}(t, \xi)=P_{n} g(t, \xi)$ for $\xi \in L_{H_{n}}^{2}$, and, finally let $W^{n}(t)$ denote the $K_{n}$-valued Wiener process defined by $W^{n}(t)=\pi_{n} w(t)$. Then, Eq. $(* 1)$ can be rewritten as

$$
\left\{\begin{array}{l}
d x^{n}(t)=\left(A^{n}\left(t, x^{n}(t)\right)+f^{n}\left(t, x_{t}^{n}\right) d t+g^{n}\left(t, x_{t}^{n}\right) d W^{n}(t)\right. \\
x^{n}(t)=\psi^{n}(t)=P_{n} \psi(t), \quad t \in[-h, 0] .
\end{array}\right.
$$

Although Eq. $(* 2)$ can be considered as an Itô stochastic differential equation in $\mathbf{R}^{n}$, we can not apply the classic results on existence and uniqueness of solutions since $A^{n}$ does not satisfy a Lipschitz type of condition. However, we can apply to this situation the results proved in the preceding section, i.e., Theorem 3 with $\|\cdot\|_{C}$ replaced by $\|\cdot\|_{L_{H}^{2}}$ in (f.2) and (g.2). Indeed, it is easy to check that $A^{n}, f^{n}, g^{n}, W^{n}$ and $\psi^{n}$ satisfy the assumptions in Theorem 3 by replacing $V, H, V^{\prime}$ by $V_{n}, H_{n}, V_{n}^{\prime}$. Therefore, for each natural number $n \geq 1$, there exists a unique $x^{n} \in I^{p}\left(-h, T ; V_{n}\right) \cap L^{2}\left(\Omega ; C\left(-h, T ; H_{n}\right)\right)$ which is the solution to $(* 2)$. Owing to the natural injections, we have that, in fact,

$$
x^{n} \in I^{p}(-h, T ; V) \cap L^{2}(\Omega ; C(-h, T ; H)) .
$$

\section{STEP 2. A priori computations}

As in the proof of Theorem 3 , we set $x^{n}:=x^{n}(s), A^{n}\left(x^{n}\right):=A^{n}\left(s, x^{n}(s)\right), f^{n}\left(x^{n}\right):=$ $f^{n}\left(s, x_{s}^{n}\right)$ and $g^{n}\left(x^{n}\right):=g^{n}\left(s, x_{s}^{n}\right)$.

The energy equality implies

$$
\begin{aligned}
\left|x^{n}(t)\right|^{2}= & \left|\psi^{n}(0)\right|^{2}+2 \int_{0}^{t}\left\langle A^{n}\left(x^{n}\right)+f^{n}\left(x^{n}\right), x^{n}\right\rangle d s \\
& +2 \int_{0}^{t}\left(x^{n}, g^{n}\left(x^{n}\right) d W^{n}(s)\right)+\operatorname{tr}\left\langle\left\langle\int_{0} g^{n}\left(x^{n}\right) d W^{n}(s)\right\rangle\right\rangle_{t}
\end{aligned}
$$


and consequently,

$$
\begin{aligned}
\left|x^{n}(t)\right|^{2}= & \left|\psi^{n}(0)\right|^{2}+2 \int_{0}^{t}\left\langle A\left(x^{n}\right)+f\left(x^{n}\right), x^{n}\right\rangle d s \\
& +2 \int_{0}^{t}\left(x^{n}, g\left(x^{n}\right) d W^{n}(s)\right)+\operatorname{tr}\left\langle\left\langle\int_{0} g\left(x^{n}\right) d W^{n}(s)\right\rangle\right\rangle_{t} .
\end{aligned}
$$

On the one hand, since

$$
\operatorname{tr}\left\langle\left\langle\int_{0}^{\cdot} g\left(x^{n}\right) d W^{n}(s)\right\rangle\right\rangle_{t}=\int_{0}^{t}\left\|g\left(x^{n}\right) \pi_{n}\right\|_{2}^{2} d s \leq \int_{0}^{t}\left\|g\left(x^{n}\right)\right\|_{2}^{2} d s,
$$

we immediately get from Condition (C) that

$$
\begin{aligned}
\left|x^{n}(t)\right|^{2} \leq & \left|\psi^{n}(0)\right|^{2}+2 \int_{0}^{t}\left\langle A\left(x^{n}\right)+f\left(x^{n}\right), x^{n}\right\rangle d s \\
& +2 \int_{0}^{t}\left(x^{n}, g\left(x^{n}\right) d W^{n}(s)\right)+\int_{0}^{t}\left\|g\left(x^{n}\right)\right\|_{2}^{2} d s \\
\leq & \left|\psi^{n}(0)\right|^{2}+\nu t+t \tau\left\|\psi^{n}\right\|_{L_{H_{n}}^{2}}^{2}+\lambda \int_{0}^{t}\left|x^{n}\right|^{2} d s \\
& \quad-\alpha \int_{0}^{t}\left\|x^{n}\right\|^{p} d s+2 \int_{0}^{t}\left(x^{n}, g\left(x^{n}\right) d W^{n}(s)\right),
\end{aligned}
$$

which, after taking expectations, yields that

$$
E\left|x^{n}(t)\right|^{2}+\alpha \int_{0}^{t} E\left\|x^{n}\right\|^{p} d s \leq E|\psi(0)|^{2}+\nu t+t \tau E\|\psi\|_{L_{H}^{2}}^{2}+\lambda \int_{0}^{t} E\left|x^{n}\right|^{2} d s .
$$

Consequently, there exist positive constants $c_{1}, c_{2}$ such that

$$
\begin{gathered}
\sup _{-h \leq t \leq T} E\left|x^{n}(t)\right|^{2} \leq c_{1} \\
E \int_{0}^{T}\left\|x^{n}(t)\right\|^{p} d t \leq c_{2},
\end{gathered}
$$

and, as $p \geq 2$, there exists $c_{3}>0$ such that

$$
E \int_{0}^{T}\left\|x^{n}(t)\right\|^{2} d t \leq c_{3}
$$

On the other hand, (42) immediately yields that

$$
\begin{aligned}
E\left[\sup _{0 \leq t \leq T}\left|x^{n}(t)\right|^{2}\right] \leq & E|\psi(0)|^{2}+\nu T+T \tau E\|\psi\|_{L_{H}^{2}}^{2}+|\lambda| \int_{0}^{T} E\left|x^{n}(t)\right|^{2} d t \\
& +2 E\left[\sup _{0 \leq t \leq T}\left|\int_{0}^{t}\left(x^{n}, g\left(x^{n}\right) d W^{n}(s)\right)\right|\right] .
\end{aligned}
$$


Evaluating the last term in (47) by applying Burkholder-Davis-Gundy's inequality (cf. see $[12]),($ G.2) and taking into account (46), we have

$$
\begin{aligned}
2 E\left[\sup _{0 \leq t \leq T}\left|\int_{0}^{t}\left(x^{n}, g\left(x^{n}\right) \pi_{n} d w(s)\right)\right|\right. & \leq 6 E\left[\left(\int_{0}^{T}\left|x^{n}\right|^{2}\left\|g\left(x^{n}\right)\right\|_{2}^{2} d s\right)^{1 / 2}\right] \\
& \leq \frac{1}{3} E\left[\sup _{0 \leq t \leq T}\left|x^{n}(t)\right|^{2}\right]+c_{4} \int_{0}^{T} E\left\|g\left(x^{n}\right)\right\|_{2}^{2} d s \\
& \leq \frac{1}{3} E\left[\sup _{0 \leq t \leq T}\left|x^{n}(t)\right|^{2}\right]+c_{4} k_{2}^{2} \int_{0}^{T} E\left\|x_{s}^{n}\right\|_{L_{V}^{2}}^{2} d s \\
& \leq \frac{1}{3} E\left[\sup _{0 \leq t \leq T}\left|x^{n}(t)\right|^{2}\right]+c_{5}+c_{6} E\|\psi\|_{L_{V}^{2}}^{2} .
\end{aligned}
$$

Hence, there exists a positive constant $c_{7}$ such that

$$
E\left[\sup _{0 \leq t \leq T}\left|x^{n}(t)\right|^{2}\right] \leq c_{7} .
$$

So, we have finally proved that

$\left\{x^{n}\right\}_{n \geq 1}$ is bounded in $I^{p}(-h, T ; V) \cap L^{2}(\Omega ; C(-h, T ; H))$,

$\left\{A\left(x^{n}\right)\right\}_{n \geq 1}$ is bounded in $L^{p^{\prime}}\left(\Omega \times(0, T) ; V^{\prime}\right)$,

$\left\{f\left(x^{n}\right)\right\}_{n \geq 1}$ is bounded in $L^{2}(\Omega \times(0, T) ; H)$,

$\left\{g\left(x^{n}\right)\right\}_{n \geq 1}$ is bounded in $L^{2}(\Omega \times(0, T) ; \mathcal{L}(K, H))$,

where $A\left(x^{n}\right), f\left(x^{n}\right), g\left(x^{n}\right)$ are defined in the obvious way and $p^{\prime}$ denotes the conjugate of $p$.

\section{STEP 3. Taking weak limits}

Owing to the last assertions in Step 2, we can ensure that there exists a subsequence $\left\{x^{n_{k}}\right\}$ of $\left\{x^{n}\right\}$ such that

$$
\begin{aligned}
& x^{n_{k}} \rightarrow x \text { in } I^{p}(-h, T ; V) \text { and weakly star in } L^{2}\left(\Omega ; L^{\infty}(-h, T ; H)\right), \\
& x^{n_{k}}(T) \rightarrow \xi \text { in } L^{2}(\Omega ; H), \\
& A\left(x^{n_{k}}\right) \rightarrow \chi \text { in } L^{p^{\prime}}\left(\Omega \times(0, T) ; V^{\prime}\right), \\
& f\left(x^{n_{k}}\right) \rightarrow \sigma \text { in } L^{2}(\Omega \times(0, T) ; H), \\
& g\left(x^{n_{k}}\right) \rightarrow \zeta \text { in } L^{2}(\Omega \times(0, T) ; \mathcal{L}(K, H)) .
\end{aligned}
$$

Let $\theta: \mathbf{R}^{1} \rightarrow \mathbf{R}^{1}$ be defined as $\theta(t)=\left\{\begin{array}{ll}0 & \text { if } t<0 \\ 1 & \text { if } t \geq 0\end{array}\right.$. If $\varphi$ is a function from $[0, T]$ into $\mathbf{R}^{1}$, we can define another function $\bar{\varphi}:(-\rho, T+\rho) \rightarrow \mathbf{R}^{1}$ (where $\rho$ is a positive fixed number) in the following way:

$$
\bar{\varphi}(t)= \begin{cases}\varphi(t) & \text { if } t \in[0, T] \\ 0 & \text { otherwise. }\end{cases}
$$


This permits us to rewrite Eq. $(* 1)$ (with $n=n_{k}$ ) as follows

$$
\begin{aligned}
\overline{\left(x^{n_{k}}(t), v_{i}\right)}= & \left(\psi(0), v_{i}\right) \theta(t)-\left(x^{n_{k}}(T), v_{i}\right) \theta(t-T) \\
& +\overline{\int_{0}^{t}\left\langle A\left(x^{n_{k}}\right)+f\left(x^{n_{k}}\right), v_{i}\right\rangle d s} \\
& +\overline{\int_{0}^{t}\left(v_{i}, g\left(x^{n_{k}}\right) \pi_{n_{k}} d w(s)\right)}, \forall t \in(-\rho, T+\rho), i=1, \ldots, n_{k} .
\end{aligned}
$$

Observe that, as the map $\phi \in L^{2}\left(\Omega \times(0, T) ; \mathcal{L}^{2}(K, H)\right) \mapsto \int_{0}^{\bullet} \phi(s) d w(s) \in L^{2}(\Omega \times(0, T) ; H)$ is linear and continuous, then it is weakly continuous (where $\mathcal{L}^{2}(K, H)$ denotes the space of all Hilbert-Schmidt operators from $K$ into $H$ ). Now, we shall prove that $g\left(x_{n_{k}}\right) \pi_{n_{k}} \rightarrow \zeta$, as $k \rightarrow \infty$, in $L^{2}\left(\Omega \times(0, T) ; \mathcal{L}^{2}(K, H)\right)$. Indeed, this convergence is equivalent to

$$
E \int_{0}^{T} \operatorname{tr}\left(Q^{*} g\left(x^{n_{k}}\right) \pi_{n_{k}}\right) d t \rightarrow E \int_{0}^{T} \operatorname{tr}\left(Q^{*} \zeta\right) d t,
$$

for all $Q \in L^{2}\left(\Omega \times(0, T) ; \mathcal{L}^{2}(K, H)\right)$, and also to

$$
E \int_{0}^{T} \operatorname{tr}\left(g\left(x^{n_{k}}\right) \pi_{n_{k}} Q\right) d t \rightarrow E \int_{0}^{T} \operatorname{tr}(\zeta Q) d t .
$$

Therefore, it is sufficient to prove that $Q \pi_{n_{k}} \rightarrow Q$ in $L^{2}\left(\Omega \times(0, T) ; \mathcal{L}^{2}(K, H)\right)$. But this is an immediate consequence of Theorem I. 2.3 in Pardoux [16].

Now, we can take weak limits in (50) and obtain:

$$
\begin{aligned}
\overline{\left(x(t), v_{i}\right)}= & \left(\psi(0), v_{i}\right) \theta(t)-\left(\xi, v_{i}\right) \theta(t-T)+\overline{\int_{0}^{t}\left\langle\chi+\sigma, v_{i}\right\rangle d s} \\
& +\overline{\int_{0}^{t}\left(v_{i}, \zeta\right) d w(s),}, \forall t \in(-\rho, T+\rho), \quad \forall i \geq 1,
\end{aligned}
$$

so it follows that

$$
\begin{gathered}
\xi=x(T) \\
d x(t)=(\chi(t)+\sigma(t)) d t+\zeta(t) d w(t), \quad t \in[0, T], \\
x(t)=\psi(t), \quad t \in[-h, 0] .
\end{gathered}
$$

Therefore, it remains to prove that $\chi+\sigma=A(x)+f(x)$ and $\zeta=g(x)$. This will be done in the next step.

STEP 4. Final step: the monotonicity method

Consider $v \in L^{p}(\Omega \times(-h, T) ; V) \cap L^{2}(\Omega \times(-h, T) ; H)$ and set

$$
\begin{aligned}
u^{n_{k}}= & -2 E \int_{0}^{T} e^{-\lambda t}\left\langle A\left(x^{n_{k}}\right)+f\left(x^{n_{k}}\right)-A(v)-f(v), x^{n_{k}}-v\right\rangle d t \\
& +\lambda E \int_{0}^{T} e^{-\lambda t}\left|x^{n_{k}}-v\right|^{2} d t-E \int_{0}^{T} e^{-\lambda t}\left\|g\left(x^{n_{k}}\right)-g(v)\right\|_{2}^{2} d t .
\end{aligned}
$$


Note that $u^{n_{k}} \geq 0$ due to Assumption (M). On the other hand, we can take limits in the terms of (54) except for the following term

$$
\begin{aligned}
y^{n_{k}}= & -2 E \int_{0}^{T} e^{-\lambda t}\left\langle A\left(x^{n_{k}}\right)+f\left(x^{n_{k}}\right), x^{n_{k}}\right\rangle d t \\
& +\lambda E \int_{0}^{T} e^{-\lambda t}\left|x^{n_{k}}\right|^{2} d t-E \int_{0}^{T} e^{-\lambda t}\left\|g\left(x^{n_{k}}\right)\right\|_{2}^{2} d t .
\end{aligned}
$$

But, (41) immediately yields that

$$
\begin{aligned}
E\left|x^{n_{k}}(t)\right|^{2}= & E\left|P_{n_{k}} \psi(0)\right|^{2}+2 E \int_{0}^{t}\left\langle A\left(x^{n_{k}}\right)+f\left(x^{n_{k}}\right), x^{n_{k}}\right\rangle d s \\
& +E\left[\operatorname{tr}\left\langle\left\langle P_{n_{k}} \int_{0} g\left(x^{n_{k}}\right) d W^{n_{k}}\right\rangle\right\rangle_{t}\right] .
\end{aligned}
$$

In particular, (56) proves that the function $t \mapsto E\left|x^{n_{k}}(t)\right|^{2}$ is absolutely continuous and hence

$$
d\left[e^{-\lambda t} E\left|x^{n_{k}}(t)\right|^{2}\right]+\lambda e^{-\lambda t} E\left|x^{n_{k}}(t)\right|^{2}=e^{-\lambda t} d\left[E\left|x^{n_{k}}(t)\right|^{2}\right] .
$$

Now, it can be obtained that

$$
\begin{aligned}
e^{-\lambda T} E\left|x^{n_{k}}(T)\right|^{2} \leq & E\left|P_{n_{k}} \psi(0)\right|^{2}-\lambda \int_{0}^{T} e^{-\lambda t} E\left|x^{n_{k}}(t)\right|^{2} d t \\
& +2 \int_{0}^{T} e^{-\lambda t} E\left\langle A\left(x^{n_{k}}\right)+f\left(x^{n_{k}}\right), x^{n_{k}}\right\rangle d t \\
& +\int_{0}^{T} e^{-\lambda t} E\left\|g\left(x^{n_{k}}\right)\right\|_{2}^{2} d t,
\end{aligned}
$$

and therefore,

$$
y^{n_{k}} \leq E|\psi(0)|^{2}-e^{-\lambda T} E\left|x^{n_{k}}(T)\right|^{2} .
$$

As an immediate consequence, it follows that

$$
\limsup _{k \rightarrow \infty} y^{n_{k}} \leq E|\psi(0)|^{2}-e^{-\lambda T} E|x(T)|^{2} .
$$

Applying Itô's formula to Eq. (52), we can get

$$
\begin{aligned}
e^{-\lambda T} E|x(T)|^{2}= & E|\psi(0)|^{2}-\lambda \int_{0}^{T} e^{-\lambda t} E|x|^{2} d t \\
& +2 \int_{0}^{T} e^{-\lambda t} E\langle\chi+\sigma, x\rangle d t+\int_{0}^{T} e^{-\lambda t} E\|\zeta\|_{2}^{2} d t .
\end{aligned}
$$

So

$$
\limsup _{k \rightarrow \infty} y^{n_{k}} \leq \int_{0}^{T} e^{-\lambda t} E\left[2\langle\chi+\sigma, x\rangle+\lambda|x|^{2}-\|\zeta\|_{2}^{2}\right] d t
$$


and finally

$$
\begin{aligned}
0 \leq \limsup _{k \rightarrow \infty} u^{n_{k}} \leq & -2 E \int_{0}^{T} e^{-\lambda t}\langle\chi+\sigma-A(v)-f(v), x-v\rangle d t \\
& +\lambda E \int_{0}^{T} e^{-\lambda t}|x-v|^{2} d t-E \int_{0}^{T} e^{-\lambda t}\|\zeta-g(v)\|_{2}^{2} d t .
\end{aligned}
$$

If we take $v=x$ in (63), it follows that $\zeta=g(x)$ and, also

$$
-2 E \int_{0}^{T} e^{-\lambda t}\langle\chi+\sigma-A(v)-f(v), x-v\rangle d t+\lambda E \int_{0}^{T} e^{-\lambda t}|x-v|^{2} d t \geq 0 .
$$

In order to finish the proof, we only need to use hemicontinuity (a.4). Indeed, we notice that the function $f$ also satisfies a similar property and it is easy to deduce from (F.2) that the map $\theta \in \mathbf{R}^{1} \mapsto(f(t, \eta+\theta \xi), x) \in \mathbf{R}^{1}$ is continuous for all $\eta, \xi \in L_{V}^{2}, x \in$ $H$ and a.e.t $\in[0, T]$. Now, in (64) setting $v=x-\theta u$ for $\theta>0$ and $u \in L^{p}(\Omega \times$ $(-h, T) ; V) \cap L^{2}(\Omega \times(-h, T): H)$, dividing by $\theta$ and letting $\theta$ tend to 0 , we then get $\forall u \in L^{p}(\Omega \times(-h, T) ; V) \cap L^{2}(\Omega \times(-h, T) ; H)$

$$
-2 E \int_{0}^{T} e^{-\lambda t}\langle\chi+\sigma-A(x)-f(x), u\rangle d t \geq 0 .
$$

Consequently, $\chi+\sigma=A(x)+f(x)$ and the proof of the theorem is complete.

\section{STABILITY OF STRONG SOLUTIONS}

In this section we shall show that under suitable conditions exponential stability can be transferred from equations without time lags to those with time lag ones. Since we are mainly interested in exponential stability problems for the second moment of solutions, we will assume there exists a process

$$
x \in I^{2}(-h, T ; V) \cap L^{2}(\Omega ; C(-h, T ; H)), \quad \forall T>0,
$$

which is the strong solution of the following problem:

$$
\left\{\begin{aligned}
d x(t) & =\left[A(t, x(t))+f\left(t, x_{t}\right)\right] d t+g\left(t, x_{t}\right) d w(t), \quad t \geq 0 \\
x(t) & =\psi(t), \quad t \in[-h, 0] .
\end{aligned}\right.
$$

In other words, $x(t)$ satisfies the following integral equation (in $V^{\prime}$ ):

$$
\begin{aligned}
x(t)= & \psi(0)+\int_{0}^{t}\left[A(s, x(s))+f\left(s, x_{s}\right)\right] d s \\
& +\int_{0}^{t} g\left(s, x_{s}\right) d w(s), \quad P-\text { a.s. }, \quad t \geq 0,
\end{aligned}
$$


and $x(t)=\psi(t), t \in[-h, 0]$. In particular, in this section we suppose all the conditions in Section 3 hold so that there exists a unique strong solution for the stochastic functional differential equation (66). For simplicity, we also suppose in the section that the coefficients $A, f$ and $g$ are continuous with respect to time $t$.

First of all, we investigate the case without hereditary characteristics. In other words, consider Eq. (67) with $h=0$ and thus $k_{1}(h)=k_{1}>0, k_{2}(h)=k_{2}>0$ in (f.2), (g.2), then the equation (66) reduces to

$$
\left\{\begin{aligned}
d x(t) & =[A(t, x(t))+f(t, x(t))] d t+g(t, x(t)) d w(t), \quad t \geq 0 \\
x(0) & =x_{0}
\end{aligned}\right.
$$

If it is possible to know the existence of some Lyapunov function, we could obtain mean square stability of solutions. Indeed, assume there exist $v \in C^{2}\left(H ; \mathbf{R}^{+}\right)$and positive constants $c_{i}, 1 \leq i \leq 4$, such that $v^{\prime}(x) \in V$ for all $x \in V$ and

$$
c_{1}|x|^{2} \leq v(x) \leq c_{2}|x|^{2}, \quad \mathbf{L} v(x) \leq-c_{3} v(x), \quad\left|v^{\prime}(x)\right| \leq c_{4}|x|,
$$

for all $x \in V$, where $\mathbf{L}$ is the associated diffusion operator defined as

$$
\mathbf{L} v(x)=\left\langle A(t, x)+f(t, x), v^{\prime}(x)\right\rangle+\frac{1}{2} \operatorname{tr}\left[v^{\prime \prime}(x) g(t, x) W g^{*}(t, x)\right], \quad \forall x \in V,
$$

we can get (applying Itô's formula to function $e^{c_{3} t} v(x), x \in H$ and Equation (68))

$$
\begin{aligned}
e^{c_{3} t} v(x(t))= & v(x(0))+c_{3} \int_{0}^{t} e^{c_{3} s} v(x(s)) d s \\
& +\int_{0}^{t} e^{c_{3} s}\left\langle A(s, x(s))+f(s, x(s)), v^{\prime}(x(s))\right\rangle d s \\
& +\int_{0}^{t} e^{c_{3} s}\left(v^{\prime}(x(s)), g(s, x(s)) d w(s)\right) \\
& \frac{1}{2} \int_{0}^{t} e^{c_{3} s} \operatorname{tr}\left[v^{\prime \prime}(x(s)) g(s, x(s)) W g^{*}(s, x(s))\right] d s .
\end{aligned}
$$

Taking expectations and observing that $\mathbf{L} v(x) \leq-c_{3} v(x)$, we have

$$
\begin{aligned}
e^{c_{3} t} E v(x(t)) & \leq E v(x(0))+c_{3} \int_{0}^{t} e^{c_{3} s} E v(x(s)) d s+\int_{0}^{t} e^{c_{3} s} E \mathbf{L} v(x(s)) d s \\
& \leq E v(x(0)),
\end{aligned}
$$

and consequently

$$
E v(x(t)) \leq e^{-c_{3} t} E v(x(0)), \quad \forall t \geq 0 .
$$

¿From the assumptions on $v$, we easily deduce that

$$
E|x(t)|^{2} \leq \frac{c_{2}}{c_{1}} e^{-c_{3} t} E|x(0)|^{2}, \quad \forall t \geq 0
$$


which means mean square exponential stability of the trivial solution of (68).

Although, as we have mentioned before, the construction of Lyapunov functions is not, in general, a trivial problem, there exists a condition that makes $v(x)=|x|^{2}$ become a natural Lyapunov function. This is the following hypothesis:

$(\mathrm{H})$ : there exists a positive constant $\gamma>0$ such that

$$
2\langle A(t, x)+f(t, x), x\rangle+\|g(t, x)\|_{2}^{2} \leq-\gamma|x|^{2}, \quad \forall x \in V
$$

Indeed, on this occasion

$$
\begin{aligned}
\mathbf{L} v(x) & =2\langle A(t, x)+f(t, x), x\rangle+\|g(t, x)\|_{2}^{2} \\
& \leq-\gamma|x|^{2}
\end{aligned}
$$

therefore, setting $c_{3}=\gamma$, we obtain exponential stability in mean square sense.

Remark. Observe that in a variety of practical situations, the following assumption $(\mathrm{H})^{\prime}$ (which seems easier to check) implies $(\mathrm{H})$ :

$(\mathrm{H})^{\prime}$ : there exists a positive constant $\alpha>0$ such that

$$
-2\langle A(t, x), x\rangle \geq \alpha\|x\|^{2}, \forall x \in V \text { and }-\alpha+2 k_{1} \beta^{2}+k_{2}^{2} \beta^{2}<0,
$$

where $k_{1}, k_{2}$ both are nonnegative constants in (f.2), (g.2) and $\beta>0$ denotes the constant satisfying

$$
|x| \leq \beta\|x\|, \quad \forall x \in V
$$

Indeed, note that

$$
\begin{aligned}
2\langle A(t, x)+f(t, x), x\rangle & +\|g(t, x)\|_{2}^{2} \\
& \leq-\alpha\|x\|^{2}+2(f(t, x), x)+\operatorname{tr}\left[g(t, x) W g^{*}(t, x)\right] \\
& \leq-\alpha\|x\|^{2}+2|f(t, x) \| x|+\operatorname{tr}\left[g(t, x) W g^{*}(t, x)\right] \\
& \leq-\alpha\|x\|^{2}+2 k_{1} \beta^{2}\|x\|^{2}+k_{2}^{2} \beta^{2}\|x\|^{2} \\
& \leq\left[-\alpha+2 k_{1} \beta^{2}+k_{2}^{2} \beta^{2}\right] \beta^{-2}|x|^{2}
\end{aligned}
$$

and denote $\gamma=\left[\alpha-2 k_{1} \beta^{2}-k_{2}^{2} \beta^{2}\right] \beta^{-2}$, the assumption $(\mathrm{H})$ holds.

In what follows, we shall show that the same hypotheses as above (mainly (f.2),(g.2) and $\left.(\mathrm{H})^{\prime}\right)$ imply mean square exponential stability of the trivial solution of the stochastic functional differential equation (66). However, it is particularly worth pointing out that on this occasion the constants $k_{1}, k_{2}$ are generally dependent on the time lag constant $h>0$. This fact simply means that in order to obtain exponential stability, the time lag must be sufficiently small. However, as will be shown by Examples 1, 2 below, on some occasions such as the time delay case, the constant $k_{1}$ or $k_{2}$ could be independent on $h>0$ so that the stability is true for any $h>0$, a result which improves that of Mao and Shah [13] in 
finite dimensional spaces. For our ends, let us firstly study some stability criteria for the stochastic functional differential equation (66).

Theorem 4. Suppose there exists a positive constant $\lambda>0$ such that for all $t \geq 0$,

$$
E\left(2<A(t, \phi(0))+f(t, \phi), \phi(0)>+\operatorname{tr}\left[g(t, \phi) W g(t, \phi)^{*}\right]\right)<-\lambda E|\phi(0)|^{2}
$$

provided $\phi=\{\phi(s):-h \leq s \leq 0\} \in C_{\mathcal{F}_{0}}^{b}([-h, 0] ; V)$ satisfying

$$
E\|\phi\|_{C}^{2} \leq e^{\lambda h} E|\phi(0)|^{2}
$$

where $C_{\mathcal{F}_{0}}^{b}([-h, 0] ; V)$ denotes the space of all $\mathcal{F}_{0}$-measurable, bounded continuous processes from $[-h, 0]$ into $V$. Then for all $\psi \in C_{\mathcal{F}_{0}}^{b}([-h, 0] ; V)$, there exists a positive constant $K \geq 1$ such that

$$
E|x(t, \psi)|^{2} \leq K \cdot \sup _{-h \leq s \leq 0} E|\psi(s)|^{2} \cdot e^{-\lambda t} \quad \text { for all } \quad t \geq 0 .
$$

Proof. Suppose (71) is not true, then there exists a $\rho \geq 0$ such that

$$
e^{\lambda t} E|x(t ; \psi)|^{2} \leq e^{\lambda \rho} E|x(\rho ; \psi)|^{2}=K \cdot \sup _{-h \leq s \leq 0} E|\psi(s)|^{2},
$$

for all $0 \leq t \leq \rho$, and there is a sequence $\left\{t_{k}\right\}_{k \geq 1}$ in $\mathbf{R}_{+}$such that $t_{k} \downarrow \rho$, as $k \rightarrow \infty$, and

$$
e^{\lambda t_{k}} E\left|x\left(t_{k} ; \psi\right)\right|^{2}>e^{\lambda \rho} E|x(\rho ; \psi)|^{2} .
$$

On the other hand, by virtue of (72) we deduce

$$
E|x(\rho+\theta ; \psi)|^{2} \leq e^{\lambda(\rho-t)} E|x(\rho ; \psi)|^{2} \leq e^{\lambda h} E|x(\rho ; \psi)|^{2},
$$

for all $-h \leq \theta \leq 0$, which, in view of the assumptions (69)(70), immediately implies that

$$
E\left(2<A(\rho, x(\rho))+f\left(\rho, x_{\rho}\right), x(\rho ; \psi)>+\operatorname{tr}\left[g\left(\rho, x_{\rho}\right) W g\left(\rho, x_{\rho}\right)^{*}\right]\right)<-\lambda E|x(\rho)|^{2} .
$$

By the continuity of the solution and the functions $f$ and $g$, we see that for some sufficiently small $h>0$,

$$
E\left(2<A(t, x(t))+f\left(t, x_{t}\right), x(t ; \psi)>+\operatorname{tr}\left[g\left(t, x_{t}\right) W g\left(t, x_{t}\right)^{*}\right]\right)<-\lambda E|x(t)|^{2},
$$

for all $t \in[\rho, \rho+h]$. Now by Itô's formula, for all sufficiently small $h>0$,

$$
\begin{aligned}
e^{\lambda(\rho+h)} & E|x(\rho+h ; \psi)|^{2}-e^{\lambda \rho} E|x(\rho ; \psi)|^{2} \\
= & \int_{\rho}^{\rho+h} e^{\lambda t}\left[\lambda E|x(t ; \psi)|^{2}+E\left(2<A(t, x(t))+f\left(t, x_{t}\right), x(t)>\right.\right. \\
& \left.\left.\quad+\operatorname{tr}\left[g\left(t, x_{t}\right) W g\left(t, x_{t}\right)^{*}\right]\right)\right] d t \\
\leq & 0 .
\end{aligned}
$$


However, this contradicts (73), so the result (71) must be true.

Theorem 5. Suppose the assumptions in Section 3 for uniqueness and existence of the strong solution of (*) hold. Assume further (f.2), (g.2) and $(H)^{\prime}$ hold, then the strong solution of $(*)$ is mean square exponentially stable. Moreover, there exists a positive constant $K \geq 1$ such that for all $t \geq 0$

$$
E|x(t, \psi)|^{2} \leq K \cdot \sup _{-h \leq s \leq 0} E|\psi(s)|^{2} \cdot e^{-\lambda t} \quad \text { for all } \quad t \geq 0 .
$$

Proof. If $t \geq 0$ and $\phi=\{\phi(s):-h \leq s \leq 0\} \in C_{\mathcal{F}_{0}}^{b}([-h, 0] ; V)$ satisfies

$$
E\|\phi\|_{C}^{2} \leq e^{\lambda h} E|\phi(0)|^{2}
$$

then by Assumptions (f.2), (g.2) and $(\mathrm{H})^{\prime}$, it is easy to deduce for all $t \geq 0$,

$$
\begin{aligned}
E(2<A(t, \phi(0)) & \left.+f(t, \phi), \phi(0)>+\operatorname{tr}\left[g(t, \phi) W g(t, \phi)^{*}\right]\right) \\
\leq & -\alpha E\|\phi(0)\|^{2}+2 k_{1} E\|\phi\|_{C}|\phi(0)|+k_{2}^{2} E\|\phi\|_{C}^{2} \\
\leq & -\alpha E\|\phi(0)\|^{2}+2 k_{1} e^{\lambda h} E|\phi(0)|^{2}+k_{2}^{2} e^{\lambda h} E|\phi(0)|^{2} \\
\leq & {\left[-\alpha+2 k_{1} \beta^{2} e^{\lambda h}+k_{2}^{2} \beta^{2} e^{\lambda h}\right] E\|\phi(0)\|^{2} . }
\end{aligned}
$$

On the other hand, by virtue of the assumption $(\mathrm{H})^{\prime}$, we have

$$
\alpha>2 k_{1} \beta^{2}+k_{2}^{2} \beta^{2} \text {. }
$$

Thus, we can choose a suitable $\lambda>0$ small enough such that

$$
0<\alpha-2 k_{1} \beta^{2} e^{\lambda h}-k_{2}^{2} \beta^{2} e^{\lambda h}
$$

which immediately implies that

$$
\begin{aligned}
E(2<A(t, \phi(0)) & \left.+f(t, \phi), \phi(0)>+\operatorname{tr}\left[g(t, \phi) W g(t, \phi)^{*}\right]\right) \\
\leq & -\left[\alpha-2 k_{1} \beta^{2} e^{\lambda h}-k_{2}^{2} \beta^{2} e^{\lambda h}\right] \beta^{-2} E|\phi(0)|^{2} .
\end{aligned}
$$

Therefore, in view of Theorem 5 the strong solution is mean square exponentially stable.

Next, as we have mentioned in Section 1, one can prove pathwise exponential stability.

Theorem 6. Under the assumptions in Theorem 5, there exist positive constants $\alpha_{1}, \alpha_{2}$ and a subset $\Omega_{0} \subset \Omega$ with $P\left(\Omega_{0}\right)=0$ such that, for each $\omega \in \Omega \backslash \Omega_{0}$, there exists a positive random variable $T(\omega)$ such that the following holds

$$
|x(t, \omega)|^{2} \leq \alpha_{1} E \sup _{-h \leq s \leq 0}|\psi|^{2} e^{-\alpha_{2} t}, \quad \forall t \geq T(\omega) .
$$


Proof. The proof follows from Itô's formula, Burkholder-Davis-Gundy's inequality (cf. see [12]) and Borel-Cantelli's lemma, using the suitable change of variables in the terms containing the time aftereffect (see, for example, Caraballo and Liu [3] and the references therein for similar results).

Remark. In the finite dimensional case, that is, when $V=H=\mathbf{R}^{n}, \beta=1, K=$ $\mathbf{R}^{n \times m}, w(t)$ is an $m$-dimensional Brownian motion and, as a consequence, $W=I$ (the identity matrix), Theorems 5 and 6 guarantee exponential stability (both mean square and pathwise) for the solutions to $(66)$ if $(\mathrm{H})^{\prime}$ are fulfilled, where $(\mathrm{H})^{\prime}$ can be rewritten now as follows:

$(\mathrm{H})^{\prime \prime}$ There exists a positive constant $\alpha$ such that

$$
-2 x^{T} A(t, x) \geq \alpha|x|^{2}, \quad \forall x \in \mathbf{R}^{n} \text { and }-\alpha+2 k_{1}+k_{2}^{2}<0,
$$

where $x^{T}$ denotes the transpose of $x$. However, under this condition the results in [13] only give exponential stability if the delay function there is $\tau(t)=t-h$ with $h$ sufficiently small.

\section{EXAMPLES}

Now, we are going to apply the results proved in the previous sections to obtain stability of stochastic differential (ordinary and partial) functional equations.

Example 1. Firstly, consider the linear stochastic differential delay equation appearing in Example 4.1 from Mao and Shah [13]:

$$
d x(t)=\left[A_{0} x(t)+B_{0} x(t-h)\right] d t+\sum_{i=1}^{m} B_{i} x(t-h) d w_{i}(t)
$$

where $A_{0}, B_{0}, B_{i}$ are all $n \times n$ matrices and $w_{i}(t)$ are mutually independent, $n$-dimensional standard Brownian motions, $1 \leq i \leq m$.

If $A_{0}$ is negative definite, that is, there exists $\alpha>0$ such that $2 x^{T} A_{0} x \leq-\alpha|x|^{2}$, and $B_{0}, B_{i}, 1 \leq i \leq m$ satisfy

$$
-\alpha+2\left\|B_{0}\right\|+\sum_{i=1}^{m}\left\|B_{i}\right\|^{2}<0
$$

then, it is easy to prove that

$$
A_{0}+B_{0}+\left(A_{0}+B_{0}\right)^{T}+\sum_{i=1}^{m} B_{i}^{T} B_{i}=-Q
$$

with $Q$ being a symmetric positive definite matrix. Consequently, we can apply the sufficient conditions in Mao and Shah [13] and, therefore, we obtain exponential stability 
when $h$ is small enough. However, since $(\mathrm{H})^{\prime \prime}$ also holds (by setting $k_{1}=\left\|B_{0}\right\|, k_{2}^{2}=$ $\sum_{i=1}^{m}\left\|B_{i}\right\|^{2}$ ), our results (see the last remark in Section 5) imply exponential stability for all $h \geq 0$, even in the more general case in which $t-h$ is replaced by a general bounded delay function $\rho(t)$. In fact, let $B_{i}=0,1 \leq i \leq m$, it is well-known from Hale [6] that if $-\alpha+2\left\|B_{0}\right\|<0$, then for any $h>0$, the solution is exponentially stable. Our results actually present a stochastic version of some of those from Hale [6].

Example 2. Consider the semilinear stochastic heat equation with finite time lags $r_{1}, r_{2}$ $\left(r>r_{1}, r_{2} \geq 0\right)$

$$
\begin{gathered}
d Z(t, x)=\left[\mu \frac{\partial^{2}}{\partial x^{2}} Z(t, x) d t+\alpha_{1} \int_{-r_{1}}^{0} Z(t+u, x) h(u) d u\right] d t+\alpha(Z(t)) Z\left(t-r_{2}, x\right) d \beta(t), \\
t \geq 0, \quad \mu>0, \quad \alpha_{1} \geq 0, \\
Z(t, 0)=Z(t, \pi)=0, \quad t \geq 0, \quad Z(s, x)=\phi(s, x), \quad \phi(\cdot, x) \in C\left([-r, 0], \mathbf{R}^{1}\right), \\
\phi(s, \cdot) \in L^{2}(0, \pi), \quad s \in[-r, 0], \quad x \in[0, \pi], \quad E\|\phi\|_{C}<\infty,
\end{gathered}
$$

where $\beta(t)$ is a standard Wiener process and $E\|\phi\|_{C}^{2}=E\left\{\sup _{-r \leq s \leq 0}\|\phi(s)\|_{H}^{2}\right\} . \alpha: \mathbf{R}^{1} \rightarrow$ $\mathbf{R}^{1}, h:\left[-r_{1}, 0\right] \rightarrow \mathbf{R}^{1}$ are two bounded, Lipschitz continuous function with $|\alpha(x)| \leq K$, $|h(u)| \leq M, x \in \mathbf{R}^{1}, u \in\left[-r_{1}, 0\right], M, K>0$. Define $V=H_{0}^{1}[0, \pi], H=L^{2}[0, \pi]$ with the corresponding boundary conditions above.

Let $A=\frac{\partial^{2}}{\partial x^{2}}$ with the domain

$$
\mathcal{D}(A)=\left\{u \in L^{2}(0, \pi), \frac{\partial u}{\partial x}, \frac{\partial^{2} u}{\partial x^{2}} \in L^{2}(0, \pi), u(0)=u(\pi)=0\right\},
$$

so it is easy to deduce

$$
2<A u, u>\leq-2 \mu\|u\|_{V}^{2}, \quad u \in V .
$$

On the other hand, it is clear that

$$
E\left\|\alpha_{1} \int_{-r_{1}}^{0} Z(t+u, \cdot) h(u) d u\right\|_{H}^{2} \leq\left(\alpha_{1} r_{1} M\right)^{2}\left\|Z_{t}\right\|_{C}^{2},
$$

we have, by a straightforward computation and applying Theorems 5, 6 to the above equation, if $2 \mu>2\left(\alpha_{1} r_{1} M\right)^{2}+K^{2}$ and for arbitrary $r_{2}>0$ the strong solution is mean square and almost sure exponentially stable.

\section{ACKNOWLEDGEMENTS}

We would like to thank Professor M. Kelbert for a helpful remark on the initial draft. The research of T. Caraballo has been partially supported by D.G.I.C.Y.T (Spain) Proyecto No. PB95-1242. One of the authors, K. Liu would also like to acknowledge the support of EPSRC Grant GR/K 70397. All the authors want to thank the referee for his/her helpful suggestions. 


\section{BIBLIOGRAPHY}

[1]. T. Caraballo, Existence and Uniqueness of Solutions for Non-Linear Stochastic Partial Differential Equations, Collect. Math. 42, 1 (1991), 51-74.

[2]. T. Caraballo, Asymptotic Exponential Stability of Stochastic Partial Differential Equations with Delay, Stochastics. 33 (1990), 27-47.

[3]. T. Caraballo and K. Liu, On Exponential Stability Criteria of Stochastic Partial Differential Equations, Stoch. Proc. Appl. 83, (1999), 289-301.

[4]. P.L. Chow, Stability of Nonlinear Stochastic Evolution Equations, J. Math. Anal. Appl., 89 (2) (1982), 400-419.

[5]. L.E. El'sgol'ts and S.B. Norkin, Introduction to the Theory and Applications of Differential Equations with Deviating Arguments, Academic Press, (1973).

[6]. J.K. Hale, Theory of Functional Differential Equations, Springer-Verlag, (1971).

[7]. A. Ichikawa, Stability of Semilinear Stochastic Evolution Equations, J. Math. Anal. Appl., 90 (1982), 12-44.

[8]. V.B. Kolmanovskii and A. Myshkis, Applied Theory of Functional Differential Equations, Kluwer Academic Publishers, (1992).

[9]. V.B. Kolmanovskii and V.R. Nosov, Stability of Functional Differential Equations, Academic Press, (1986).

[10]. N.N. Krasovskii, Stability of Motions, Stanford University Press, (1963).

[11]. H.J. Kushner, On the Stability of Process Defined by Stochastic DifferenceDifferential Equations, J. Differential Eqns. 4 (1968), 424-443.

[12]. X.R. Mao, Exponential Stability of Stochastic Differential Equations, Marcel Dekker, (1994).

[13]. X.R. Mao and A. Shah, Exponential Stability of Stochastic Differential Delay Equations, Stochastics. 60 (1997), 135-153.

[14]. M. Métivier and J. Pellaumail, Stochastic Integration, Academic Press, New York, (1980).

[15]. S.E.A. Mohammed, Stochastic Functional Differential Equations, Longman Scientific and Technical, (1986).

[16]. E. Pardoux, Equations aux Dérivées Partielles Stochastiques Nonlinéaires Monotones, Thesis, Université Paris Sud, (1975).

[17]. E. Pardoux, Stochastic Partial Differential Equations and Filtering of Diffusion Processes, Stochastics. 3 (1979), 127-167. 